\title{
Effects of the Mean Flow on Martian Transient Eddy Activity: Studies with an Idealized General Circulation Model
}

\author{
TODD A. MOORING ${ }^{\mathrm{a}}$ \\ Program in Atmospheric and Oceanic Sciences, Princeton University, Princeton, New Jersey \\ ISAAC M. HELD AND R. JOHN WILSON ${ }^{\mathrm{b}}$ \\ NOAA/Geophysical Fluid Dynamics Laboratory, Princeton, New Jersey
}

(Manuscript received 17 August 2018, in final form 14 May 2019)

\begin{abstract}
The extent to which the eddy statistics of the Martian atmosphere can be inferred from the mean state and highly simplified assumptions about diabatic and frictional processes is investigated using an idealized general circulation model (GCM) with Newtonian relaxation thermal forcing. An iterative technique, adapted from previous terrestrial studies, is used to generate radiative equilibrium temperatures such that the threedimensional time-mean temperature fields of the idealized model match means computed from the Mars Analysis Correction Data Assimilation (MACDA). Focusing on a period of strong Northern Hemisphere eddy activity prior to winter solstice, it is found that the idealized model reproduces some key features of the spatial patterns of the MACDA eddy temperature variance and kinetic energy fields. The idealized model can also simulate aspects of MACDA's seasonal cycle of spatial patterns of low-level eddy meridional wind and temperature variances. The most notable weakness of the model is its eddy amplitudes-both their absolute values and seasonal variations are quite unrealistic, for reasons unclear. The idealized model was also run with a mean flow based on output from the Geophysical Fluid Dynamics Laboratory (GFDL) full-physics Mars GCM. The idealized model captures the difference in mean flows between MACDA and the GFDL Mars GCM and reproduces a bias in the more complex model's eddy zonal wavenumber distribution. This implies that the mean flow is an important influence on transient eddy wavenumbers and that improving the GFDL Mars GCM's mean flow would make its eddy scales more realistic.
\end{abstract}

\section{Introduction}

One of the enduring questions of large-scale atmospheric dynamics is which aspects of eddy phenomena in a planetary atmosphere can be understood primarily as functions of the time-mean flow. The results of the classic linear instability problems of Eady and Charney show that at least some eddy properties can be understood in this manner (Chang et al. 2002), and it is natural to wonder how far such an approach can be pushed-for example, can spatial and temporal variations of eddy intensity be explained by the mean

\footnotetext{
${ }^{a}$ Current affiliation: Department of the Geophysical Sciences, University of Chicago, Chicago, Illinois.

${ }^{\mathrm{b}}$ Current affiliation: NASA Ames Research Center, Moffett Field, California.
}

Corresponding author: Todd A. Mooring, tmooring@alum.mit.edu state with minimal reference to the details of damping processes?

A vast literature has developed around such questions in the context of the terrestrial atmosphere. The associated hierarchy of models ranges from linear instability analyses of realistic zonally averaged (e.g., Valdes and Hoskins 1988) or even three-dimensional (e.g., Frederiksen 1983) mean flows to idealized general circulation models (GCMs) with simplified forcing but relatively realistic mean states (e.g., Lunkeit et al. 1998; Hall 2000; Chang 2006) to fullphysics atmosphere and coupled models (e.g., Blackmon and Lau 1980; Chang et al. 2012; Harvey et al. 2012).

Although the existence of transient eddies on Mars has been known for decades (e.g., Leovy et al. 1972; Ryan et al. 1978; Barnes 1980) they have naturally received less study than their Earthly counterparts. The two end members of the eddy model hierarchy, linear instability calculations (Mintz 1961; Leovy 1969; Blumsack and Gierasch 1972; Gadian 1978; Barnes 1984; Tanaka and Arai 1999) and 
full-physics Mars GCMs (e.g., Leovy and Mintz 1966, 1969; Barnes et al. 1993; Hollingsworth et al. 1996), have existed for more than half a century but the intermediate levels are less well explored.

A handful of idealized nonlinear models of Martian transient eddies have been published since the mid-1990s, but the effects of topography and stationary waves on the transient eddies have seen only limited investigation with such models (Collins and James 1995; Keppenne and Ingersoll 1995; Haberle et al. 1997; Matheson 2000). Two of these studies (Collins and James 1995; Haberle et al. 1997) largely neglected such effects by primarily or exclusively using flat topography and zonally symmetric radiative forcing for their GCMs. In contrast Keppenne and Ingersoll (1995) focused on the effect of topography on transient eddies but used a shallow water model and thus unrealistically restricted the eddies to being purely barotropic. Matheson (2000) used several different topographies in his GCM study of interhemispheric differences in eddy amplitude but apparently ignored zonal variations of the eddy properties themselves. A number of additional studies have used full-physics Mars GCMs to examine topographic effects, but again sometimes ignore the zonal structure of eddy fields (e.g., Barnes et al. 1993; Hollingsworth et al. 1996; Mulholland et al. 2016).

A simplified model that can be used to explore the relationship between three-dimensional Mars-like mean flows and transient eddy activity is worth constructing, because zonal modulation is clearly present in the Martian topography (e.g., Smith et al. 2003), in the time-mean state of the atmosphere (e.g., Banfield et al. 2003; Hinson et al. 2003), and in the observed eddy statistics themselves (e.g., Banfield et al. 2004; Hinson et al. 2012). The idealized GCM we created requires information about the mean flow (specifically, the three-dimensional time-mean temperature field) as input, which we take from reanalysis or a full-physics Mars GCM. These datasets and the idealized GCM itself are described in section 2. Our main results are presented in section 3. First, we extensively evaluate the idealized GCM's performance for a single season in section 3a. Its ability to reproduce the seasonal cycle of eddy activity, including a solstitial pause described by Lewis et al. (2016) and Mulholland et al. (2016), is examined in section 3b. Finally, in section $3 c$ the idealized GCM is used to investigate the cause of an eddy wavenumber bias found in the full-physics Mars GCM. A discussion and conclusions are presented in section 4 .

\section{Data and methods}

\section{a. Mars reanalysis and full-physics GCM}

Most of the idealized GCM simulations used in this paper have mean flows derived from the Mars Analysis Correction Data Assimilation (MACDA), which is primarily described by Montabone et al. (2014). It is based on two types of data, both from the Thermal Emission Spectrometer (TES) instrument on Mars Global Surveyor: nadir temperature profiles and total column dust opacities. The underlying model is the U.K. Mars GCM (Forget et al. 1999) with an updated topography dataset (Lewis et al. 2007). To create MACDA, the model was run at T31 spectral resolution. The final dataset is publicly available at a $5^{\circ}$ horizontal resolution on the model's 25 sigma levels with a temporal resolution of 12 times per sol. (A sol is a Martian solar day, $\sim 1.03$ Earth days.)

Seasons on Mars are described in terms of solar longitude $L_{s}$, the angle between the line connecting Mars and the sun and the line connecting the sun and the position of Mars at its northern spring equinox (Allison and McEwen 2000), and recent Mars atmospheric science literature generally numbers Mars years according to the scheme of Clancy et al. (2000). In these terms the temporal extent of MACDA is approximately Mars year (MY) $24, L_{s}=141^{\circ}$ to MY27, $L_{s}=86^{\circ}$, or the $\sim 2.8$ Mars years extending from May 1999 to September 2004 (LMD Mars Climate Database Team 2010). However, TES data are not continuously available during this interval and some of the data availability gaps are longer than the time scale over which the model can effectively retain the information in assimilated temperature data (Montabone et al. 2014). Only one such gap is of importance to the present investigation-MY26, $L_{s}=202^{\circ}-205^{\circ}$ (Mooring and Wilson 2015).

We also describe the results of idealized GCM experiments with mean flows based on a simulation performed with the Geophysical Fluid Dynamics Laboratory (GFDL) full-physics Mars GCM. This simulation was previously described by Kavulich et al. (2013) and employed the GFDL finite-volume dynamical core with a C22 $\left(\sim 4^{\circ}\right)$ resolution cubed-sphere grid in the horizontal (Lin 2004; Putman and Lin 2007; Zhao et al. 2009) and 36 terrain-following hybrid sigma-pressure levels. Although the dynamical fields of the model are allowed to freely evolve in response to forcing from the model's physical parameterizations, total column dust opacities are constrained to follow a zonally averaged form of the opacity dataset created by Montabone et al. (2011) from TES MY24 and 25 observations (i.e., some of the same dust observations used in the creation of MACDA). Model output from the GFDL Mars GCM simulation is available four times per sol for more than 2 Mars years. Both years are forced with the same seasonally varying dust scenario and differ only in their initial conditions and (slightly) in assumed total masses of $\mathrm{CO}_{2}$, the main atmospheric constituent. 


\section{b. A Mars-like idealized GCM}

Our idealized GCM is essentially a Martian version of one created by Chang (2006). It uses the GFDL spectral dynamical core (Held and Suarez 1994), forced by Newtonian relaxation to a three-dimensional radiative equilibrium temperature field $T_{\text {eq }}$. The only other major physics parameterization is a Rayleigh friction surface boundary layer active for $\sigma \geq 0.7-$ a convection parameterization is not used, consistent with similar terrestrial models (Held and Suarez 1994). Basic input parameters of the model, such as the planetary radius, rotation rate, and thermodynamic properties of the atmospheric gas, have been chosen to be appropriate to Mars. A realistic Martian topographic map is used, part of which is shown in Fig. 1.

Horizontal resolution is T42 $\left(\sim 2.8^{\circ}\right)$, which should be adequate for resolving extratropical transient eddies with typical Mars-like zonal wavenumbers of 1-4 (e.g., Banfield et al. 2004; Mooring and Wilson 2015). The vertical structure is 28 unevenly spaced sigma levels. To improve its numerical stability, the model includes an artificial horizontal diffusion on the zonally asymmetric components of the wind field in the top five levels. In more physical terms, this sponge layer begins at an altitude of $\sim 5$ scale heights, well above the levels for which we actually examine the model output. This sponge is not thought to distort the aspects of the model's behavior in which we are interested-rerunning one of our simulations with the same $T_{\text {eq }}$ field but the sponge approximately tripled in strength and its bottom extended down to $\sim 3.9$ scale heights (seven levels) had little effect on the results. The model uses a $\nabla^{8}$ hyperdiffusion at all levels.

\section{1) Generating the Relaxation TEMPERATURE FIELD}

The key to creating a realistic mean flow in the model is the technique used to generate the relaxation temperature field $T_{\text {eq. }}$. We follow Chang (2006) in using an iterative method for producing a suitable $T_{\text {eq }}$ field (cf. Lunkeit et al. 1998). The reader is referred to his paper for a more detailed exposition-here we will simply state the algorithm and describe a few technical differences of our work from Chang's.

Suppose that we want the model to reproduce a given time-independent (i.e., without a seasonal or diurnal cycle) target temperature climatology $T_{c}$. If we have a model run forced with a time-independent relaxation temperature field $T_{\mathrm{eq}}^{i}$ that produced a time-mean output temperature field $T^{i}$ we can define $T_{\text {eq }}^{i+1}$, a new $T_{\text {eq }}$ field that will hopefully yield a $\bar{T}^{i+1}$ more similar to $T_{c}$, according to

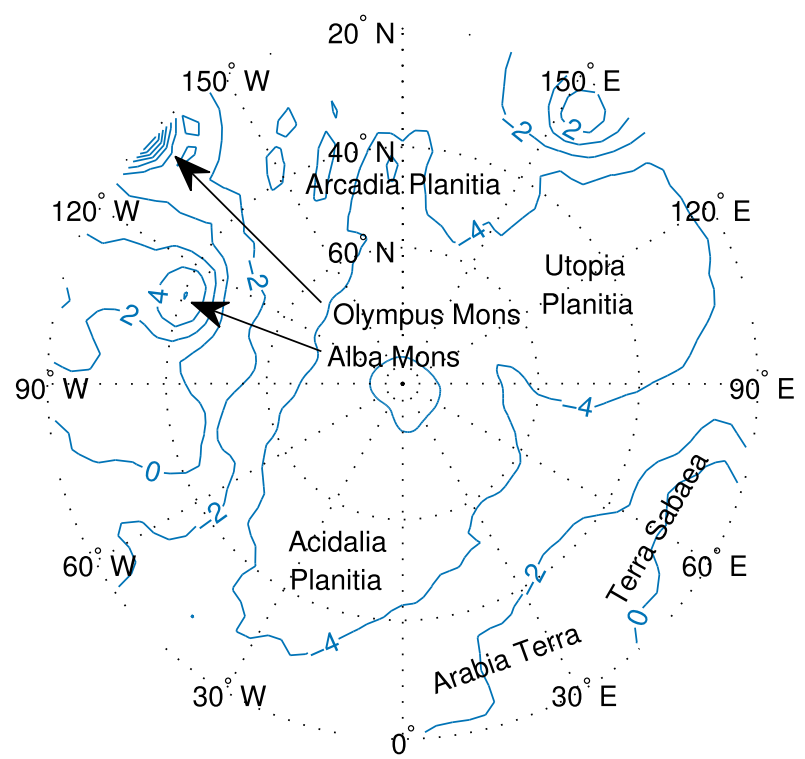

FIG. 1. Topographic map of Mars poleward of $20^{\circ} \mathrm{N}$, with heights given in kilometers relative to an areoid. Data are from the Mars Orbiter Laser Altimeter (MOLA; Smith et al. 2003). For use in the idealized GCM it has been averaged to the $64 \times 128$ grid of the model's dynamical core and spectrally truncated. Place names are generally taken from USGS Astrogeology Science Center (2015) and IAU Working Group for Planetary System Nomenclature (2001). The mountains Alba and Olympus Mons are parts of a larger elevated region, the Tharsis bulge, that is centered near $100^{\circ} \mathrm{W}$ and extends through $\sim 80^{\circ}$ of longitude.

$$
T_{\mathrm{eq}}^{i+1}=T_{\mathrm{eq}}^{i}+\alpha\left(T_{c}-\bar{T}^{i}\right),
$$

where $0<\alpha<1$. (The choice of $\alpha<1$ is intended to avoid overshooting the optimal $T_{\text {eq. }}$.) Equation (1) is then iterated until some $T_{\text {eq }}^{N}$ that yields a $\bar{T}^{N}$ sufficiently close to $T_{c}$ is generated. As will be shown later, we found this iterative technique to be quite effective when using $\alpha=1 / 3$ and defining the $\bar{T}^{i}$ as means over the final 60 sols of 100 -sol model runs. The effect of seasonal variations in the mean state is examined by simply defining a unique $T_{c}$ and generating an associated $T_{\text {eq }}$ field separately for each interval of interest-the model does not have a continuous seasonal cycle (cf. Wu and Reichler 2018).

During the iteration process model output was sampled once per sol and interpolated from the model's sigma coordinates to the pressure coordinates on which $T_{c}$ was defined before computing time means. Each new model run was initialized from the end of the previous run-in other words, the model state varied smoothly over the full length of the iteration process but the $T_{\mathrm{eq}}$ field changed discontinuously every 100 sols. The iteration process requires an initial guess of $T_{\text {eq }}$-we simply chose $T_{\mathrm{eq}}^{1}=T_{c}$. Generating final $T_{\mathrm{eq}}$ fields to force the simulations used in this paper required 13-30 100-sol model runs per simulation. 
TABLE 1. Basic information about the segments of MACDA and the GFDL Mars GCM simulation used to define target climatologies for the idealized GCM experiments. The last three rows of the table list the temporal means and extremes of global-mean surface pressure values computed for all time steps in the identified time intervals. They have been rounded to the nearest 1 Pa for listing here, although greater precision was used when setting the atmospheric masses of the idealized GCM experiments.

\begin{tabular}{lcccc}
\hline \hline & MPRE & MPAUSE & MPOST & GPRE \\
\hline Data source & MACDA & MACDA & MACDA & GFDL Mars GCM \\
Mars year & 24 & 24 & $25-49$ & 1 \\
$L_{s}$ range $\left({ }^{\circ}\right)$ & $195.35-235$ & $265.25-305$ & $621 / 12$ & $195.35-234.8$ \\
Length $($ sols $)$ & 63 & 63 & 619 & 63 \\
Mean $p_{s}(\mathrm{~Pa})$ & 602 & 620 & 606 & 608 \\
Minimum $p_{s}(\mathrm{~Pa})$ & 561 & 667 & 631 & 564 \\
Maximum $p_{s}(\mathrm{~Pa})$ & 641 & & 649 \\
\hline
\end{tabular}

\section{2) RADIATIVE AND FRICTIONAL DAMPING TIMES}

Given the above prescription for computing a suitable $T_{\text {eq }}$ field given $T_{c}$, the other major input parameters to the idealized GCM are the radiative damping time $\tau_{R}$, the Rayleigh frictional damping time $\tau_{F}$, and the frictional boundary layer top $\sigma_{b}$. Although it would arguably have been better to use a spatially varying radiative damping time (Nayvelt et al. 1997; Matheson 2000), for simplicity we used a single radiative damping time for each simulation and conducted multiple simulations with each target climatology to assess sensitivity of the results to radiative damping time. The simulations discussed in this paper use damping times of $0.5-2$ sols - similar to or shorter than the values given by Nayvelt et al. (1997, their Fig. 3) and Eckermann et al. (2011, their Fig. 4). Our choices are also similar to those of Haberle et al. (1997), who found that spatially constant radiative damping times of 1 or 2 days resulted in good agreement between their idealized Marslike GCM and a version of the NASA Ames Mars GCM, depending on the dust loading in the full-physics model.

The Rayleigh drag surface friction parameterization in the model is

$$
\left.\frac{\partial \mathbf{u}}{\partial t}\right|_{F}=-\max \left(0, \frac{\sigma-\sigma_{b}}{1-\sigma_{b}}\right) \frac{\mathbf{u}}{\tau_{F}},
$$

where $\partial \mathbf{u} /\left.\partial t\right|_{F}$ is the horizontal wind velocity tendency due to surface friction, $\tau_{F}$ is the surface value of the frictional damping time, and $\sigma_{b}$ is the top of the layer in which friction is nonzero. We opted not to conduct extensive tests of the sensitivity of results to $\tau_{F}$, using a value of $1 \mathrm{sol}$ for most of the experiments discussed in this paper. Specified or implied values of $\tau_{F}$ have varied widely among previous Mars-like atmosphere models (Zalucha and Gulbis 2012).

Although our choice of boundary layer top $\left(\sigma_{b}=0.7\right)$ is consistent with several previous terrestrial studies (Chang 2009; Held and Suarez 1994) and thinner boundary layers have sometimes been used for Mars-like idealized GCMs (e.g., Lewis et al. 1996), the actual Martian boundary layer can be up to $\sim 10 \mathrm{~km}$ deep during the day—similar to the scale height (Petrosyan et al. 2011). However a spatially and temporally uniform boundary layer thickness is the simplest possible formulation, in keeping with our goals for this study. By describing the strengths and limitations of the resulting simulations we hope to stimulate further work with models that are conceptually similar but with less radically simplified parameterizations of diabatic and frictional processes.

\section{c. Production of target climatologies}

The final piece of input needed by the idealized GCM is a target climatology, the $T_{c}$ field discussed in the previous subsection. From the data sources described in section 2 a we generated four target climatologies for use in the main body of this study: three from different seasons of MACDA and the fourth from the GFDL Mars GCM simulation. The target climatologies' properties are summarized in Table 1 and explained more thoroughly in this subsection.

We began our investigation with a target climatology based on MACDA MY24, $L_{s}=195.35^{\circ}-235^{\circ}$, which we refer to as MPRE because it is part of the PRE season of strong eddy activity prior to northern winter solstice defined by Mooring and Wilson (2015). This seasonal interval was selected because during it the Northern Hemisphere eddy wavenumber spectrum of the GFDL Mars GCM simulation is conspicuously skewed toward wavenumber 2 relative to the (presumably more realistic) spectrum of MACDA (Kavulich et al. 2013). The target climatology based on the GFDL Mars GCM simulation covers essentially the same $L_{s}$ range and is referred to as GPRE.

The two other target climatologies, referred to as MPAUSE (MY24, $L_{s}=265.25^{\circ}-305^{\circ}$ ) and MPOST (MY25, $L_{S}=20^{\circ}-49^{\circ}$ ), are named after the PAUSE and POST seasons of Mooring and Wilson (2015) and were created to test the idealized GCM's ability to reproduce the seasonal cycle of Northern Hemisphere transient eddy activity seen in MACDA. Low-level eddy activity is observed to be suppressed near winter solstice $\left(L_{s}=270^{\circ}\right)$, defining a solstitial pause (Lewis et al. 2016) that gives PAUSE its name, and 
then rebounds afterward during POST before trailing off into summer. MPAUSE is located within the solstitial pause, while MPOST is near the end of the postsolstice eddy season (Mooring and Wilson 2015).

Temperature observations to constrain MACDA were continuously available during the MY24 and 25 time periods used to generate MPRE, MPAUSE, and MPOST, which were chosen to be nearly the same length to facilitate fair comparison of idealized GCM simulations to the associated reanalysis or Mars GCM datasets. MACDA is less well constrained in the polar night than at other latitudes, because of the choice to not assimilate parts of TES profiles that appear to be supersaturated (Montabone et al. 2014), although the spreading of information by the model presumably helps to ameliorate this problem. In the vertical, TES profiles generally extend high enough to constrain the atmospheric state up to at least $\sim 0.25 \mathrm{hPa}$, above the top of the region in which we are interested (Lewis et al. 2007; L. Montabone 2019, personal communication).

Some comments are needed on the inclusion (or lack thereof) of various physical processes in the definitions of the target climatologies. As noted in the previous section there is no diurnal cycle in radiative forcing, which could conceivably affect the transient eddy activity (Collins et al. 1996; cf. Read et al. 2015). Although both MACDA and the GFDL Mars GCM used observationally based timedependent dust fields, neither model used a water ice cloud parameterization. This means that the potentially substantial effects of such clouds (Wilson 2011; Mulholland et al. 2016) will not be included in the GPRE target climatology-however, they should still influence MPRE and the other MACDA-based target climatologies via their effect on the observed temperatures assimilated into MACDA.

Furthermore, Mars is sufficiently cold that $\mathrm{CO}_{2}$ can freeze in some regions and seasons and thus release latent heat. Methods used for parameterizing latent heating in earlier models include a temperature floor (Haberle et al. 1997) or ad hoc reduction of the static stability of the target climatology (Chang 2006)—we do not include any such parameterizations in our model. Despite their lack of an explicit latent heating parameterization, the idealized GCM simulations do account for the annual cycle in atmospheric mass caused by $\mathrm{CO}_{2}$ deposition and sublimation by having their (time independent) atmospheric masses set to reproduce the space-time mean surface pressures given in the fifth row of Table 1.

\section{Results}

\section{a. Major mean and eddy fields}

To assess the overall performance of the idealized GCM we will focus on simulations using the MPRE target climatology. This subsection includes our most thorough examination of eddy field structures and evaluations of the effects of different values of $\tau_{R}$ and $\tau_{F}$. For convenience we have assigned the simulations names of the form TCLIMr $X \mathrm{f} Y$ where TCLIM is the name of the target climatology as given in Table 1 and $X$ and $Y$ are the values of the radiative and frictional damping times in sols-under this convention, the simulations used in this section are MPREr2f1, MPREr1f1, MPREr0.5f1, and MPREr1f0.5. A fifth simulation, MPREr0.5f0.5, was found to have virtually no eddy activity - evidently the mean flow was stabilized by the very short damping times. Because the spatial pattern of the remaining weak eddy activity bore little resemblance to the other simulations, we consider MPREr0.5f0.5 very unrealistic and do not include it in our figures or discussions. For all simulations output was recorded as instantaneous values four times per sol.

\section{1) TIME-MEAN FLOW}

We will begin by analyzing how well the idealized GCM can reproduce the time-mean, zonal-mean flow. Results for zonal wind and surface pressure are shown in Fig. 2. For all four combinations of $\tau_{R}$ and $\tau_{F}, T_{\text {eq }}$ fields can be generated to give the idealized GCM an extratropical zonal wind distribution much like that of the underlying MACDA dataset. However the idealized model's tropical easterlies are systematically deeper than those of MACDA, although this is not expected to significantly affect our study. Time means for the idealized GCM simulations are computed over periods of 270 sols, which virtually eliminates internal variability. Time means for MACDA are shown for both MY24 and 26-MY26 is more appropriate than MY25 for comparison to MY24 because a global dust storm took place in MY25 and overlapped the MPRE and part of the MPAUSE intervals (e.g., Mooring and Wilson 2015). The MACDA results suggest that the 270-sol segments of each idealized GCM simulation agree better with each other than do two different Mars years of the same season of MACDA. This result is more evident in the zonal-mean temperature field (Mooring 2016).

Although all of the idealized GCM simulations exhibit strong jets in the winter hemisphere, thermal wind balance does not constrain surface winds and notable differences between experiments are seen. The strongest time-mean, zonal-mean surface zonal winds exceed $20 \mathrm{~m} \mathrm{~s}^{-1}$ for MPREr2f1 and $10 \mathrm{~m} \mathrm{~s}^{-1}$ for MPREr1f1, but do not reach $10 \mathrm{~m} \mathrm{~s}^{-1}$ in any other experiment. Furthermore, the northern zero wind contour reaches the ground at much higher latitudes for MPREr2f1 and MPREr1f1 than for the other experiments. These surface wind strength differences are qualitatively consistent with geostrophic balance (approximately valid for Mars; 

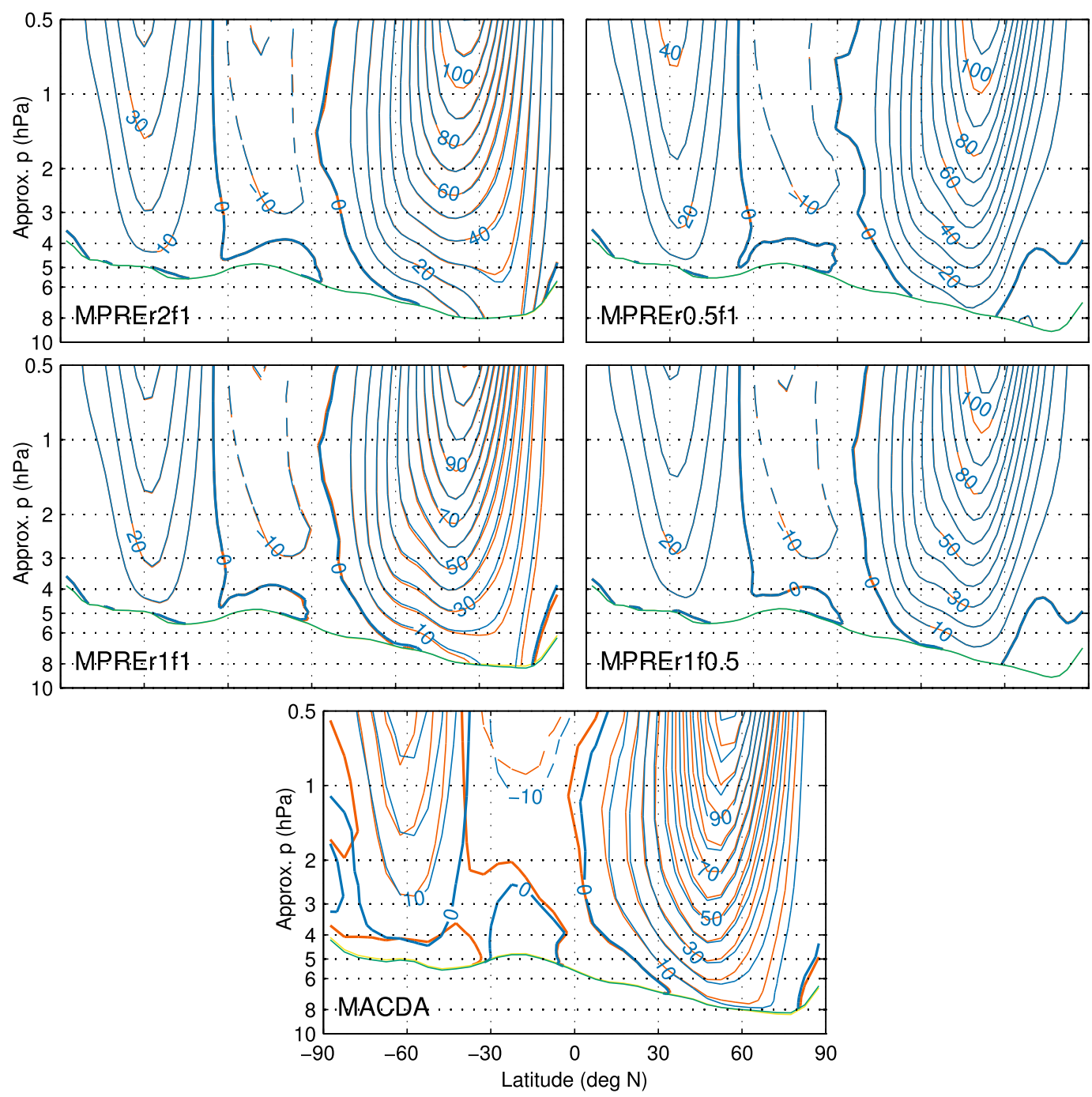

FIG. 2. Time-mean, zonal-mean zonal winds for (top),(middle) the MPRE idealized GCM experiments and (bottom) the associated segment of MACDA. Contour spacing is $10 \mathrm{~m} \mathrm{~s}^{-1}$. For the idealized GCM simulations, the blue and red lines represent time means over separate 270 -sol chunks of data to enable assessment of internal variability. For MACDA, the blue contours are a time mean over the part of MY24 used to define the MPRE target climatology and the red contours are for the same $L_{s}$ range in MY26 (excluding the $L_{s}=202^{\circ}-205^{\circ}$ TES retrieval gap). Zonal means are computed on sigma surfaces, but to portray the large Martian topography in the plots the sigma values are converted to latitude-dependent nominal pressures by multiplying by the time-mean, zonal-mean surface pressure. The green and yellow lines are surface pressures for the time intervals with zonal winds contoured in blue and red, respectively.

Leovy 2001) and the modeled distribution of zonalmean surface pressures-the pressure gradients over $\sim 60^{\circ}-75^{\circ} \mathrm{N}$ are notably less positive for MPREr2f1 and MPREr1f1 than for the other experiments. Comparing the idealized GCM experiments to MACDA, it thus appears that MPREr2f1 and MPREr1f1 have the most realistic low-level jet shapes, strengths, and surface pressure fields. Although the lowest-level mean winds in MACDA do not exceed $5 \mathrm{~m} \mathrm{~s}^{-1}$ (not shown), this may simply be the result of MACDA's more realistic boundary layer scheme or higher vertical resolution at very low levels. In principle there may also be an influence from the lower resolution of MACDA's topographythe idealized model's topography has not been truncated beyond the T42 implied by its resolution, whereas the U.K. Mars GCM topography necessarily excludes features not representable at T31 resolution.

Since a major purpose of this study is to assess the behavior of the transient eddies on realistic fully threedimensional mean flows, we also evaluated the zonally 
asymmetric components of the idealized GCM's mean states. Figure 3, which shows temperature stationary waves at $5.25 \mathrm{hPa}$, is a sample of our findings. Figure 3 demonstrates that all four idealized GCM experiments have inherited the basic structure found in MACDA, an essentially wavenumber- 2 pattern with the warm phases located above the raised topography of Alba Mons and Arabia Terra/Terra Sabaea. However, the stationary waves in different idealized GCM experiments are generally distinguishable from each other and from those in MACDA. Examination of vertical sections of the temperature stationary waves at $57.2^{\circ} \mathrm{N}$ in the idealized GCM experiments and at $57.5^{\circ} \mathrm{N}$ in MACDA shows that they are basically equivalent barotropic in all cases and that the phase relationships between the $\sim 57^{\circ} \mathrm{N}$ meridional wind and temperature stationary waves are consistent with geostrophic balance (Mooring 2016).

\section{2) ZONAL-MEAN EDDY FIELDS}

The results presented above show that the Chang (2006) technique for creating a specified realistic mean flow works in the Martian context. We will thus proceed to study the idealized GCM simulations' transient eddy fields, starting with their zonal-mean meridional-vertical structures. Because the time-mean state was found to have a nonnegligible dependence on the exact values of $\tau_{R}$ and $\tau_{F}$, we will continue to make relevant sensitivity tests. We focus our analyses of MPRE eddy activity on $T^{\prime 2}$ and eddy kinetic energy (EKE), with EKE formally defined as $(1 / 2)\left(\overline{u^{\prime 2}}+\overline{v^{\prime 2}}\right)$ - further results on $\overline{v^{\prime} T^{\prime}}$ and $\overline{u^{\prime} v^{\prime}}$ are presented in Mooring (2016). Transient eddies, denoted by the $(\cdot)^{\prime}$ operator, were extracted from MACDA and the idealized GCM simulations using a bandpass filter with half power points at 1.24 and 15.36 sols. This is adequate to contain the low-level transient eddies found in MACDA while removing thermal tides.

Figure 4 shows the $T^{\prime 2}$ fields for the MPRE idealized GCM simulations. They exhibit clear maxima in the midlatitude lower atmosphere. Inspection of the underlying data reveals that the single largest zonal-mean $\overline{T^{\prime 2}}$ value for both segments of each of the four experiments is located at $51.6^{\circ} \mathrm{N}$ on the lowest model level $(\sigma=$ 0.9966). Values of these maxima are listed in Table 2. The results suggest that increasing $\tau_{R}$ while $\tau_{F}$ is held fixed acts to increase eddy amplitudes. This is intuitively reasonable - the Newtonian relaxation damps the eddies (Chang 2006) and does so more strongly for shorter $\tau_{R}$, while (adiabatic) linear baroclinic growth rates should be similar for all of the experiments because of the relatively fixed mean flows. By comparing the results for MPREr1f1 and MPREr1f0.5, we see that reducing $\tau_{F}$ at fixed $\tau_{R}$ may increase the eddies' intensity. Although counterintuitive, this is perhaps a manifestation of the barotropic governor (James and Gray 1986). This reduction of eddy intensity with increasing $\tau_{F}$ at fixed $\tau_{R}$ is also found for $\overline{v^{\prime} T^{\prime}}$, although the intensification with increasing $\tau_{R}$ at fixed $\tau_{F}$ is not (Mooring 2016).

The spatial patterns of zonal-mean $\overline{T^{\prime 2}}$ are in reasonable agreement with those found in MACDA, which features basically the same low-level local maximum. MACDA also features a separate local maximum higher in the atmosphere, which is most prominently reproduced by MPREr2f1. (The other three simulations have such maxima as well but for MPREr0.5f1 and MPREr1f0.5 they are not visible at the contour resolution used in Fig. 4.) In MY24, the maximum of zonal-mean $\overline{T^{\prime 2}}$ is located at nearly the same latitude as the idealized GCM maxima $\left(52.5^{\circ} \mathrm{N}\right)$ but a higher level $(\sigma=0.8312)$. Note the reduction in zonal-mean $\overline{T^{\prime 2}}$ at very low levels, analogous to the zonal wind reduction documented in Fig. 2.

In MY26, the largest zonal-mean $\overline{T^{\prime 2}}$ values in the upper local maximum region are actually larger than those in the lower local maximum region, but the lower region remains prominent. It peaks at the exact same latitude and level as the MY24 maximum (Table 2). Thus although the idealized GCM is fairly successful in reproducing the spatial structure of the MACDA zonalmean $\overline{T^{\prime 2}}$ field, peak amplitudes in the lower local maximum region are consistently excessively large. This bias may be concentrated at the very lowest levelscompare for example the $30-\mathrm{K}^{2}$ contours for MACDA and the idealized GCM experiments.

Eddy kinetic energies are shown in Fig. 5. The most prominent aspect of the MACDA zonal-mean EKE field is its concentration of eddy activity near the top of the plotted region. This feature is present in some form in all four of the idealized GCM simulations, albeit not necessarily with a realistic amplitude or meridional extent. MPREr2f1 stands out in producing a local maximum very close to the pole at $\sim 2 \mathrm{hPa}$, which has no evident counterpart in MACDA. As for $T^{\prime 2}$, the idealized GCM simulations succeed in capturing a major feature of the spatial distribution of eddy activity but do not portray its amplitude very accurately.

\section{3) HORIZONTAL STRUCTURE OF EDDY FIELDS}

We now evaluate the latitude-longitude structure of the same two eddy fields. To limit the scope of the analysis, each field is evaluated at only a single level: $\sigma \approx$ 0.9 for $\overline{T^{\prime 2}}$ and $\sigma \approx 0.5$ for EKE. The value $\sigma \approx 0.9$ was chosen for $\overline{T^{\prime 2}}$ to capture its low-level maximum, while the EKE maximum is generally at higher altitudes and so $\sigma \approx 0.5$ represents a compromise between capturing 
MPREr2f1

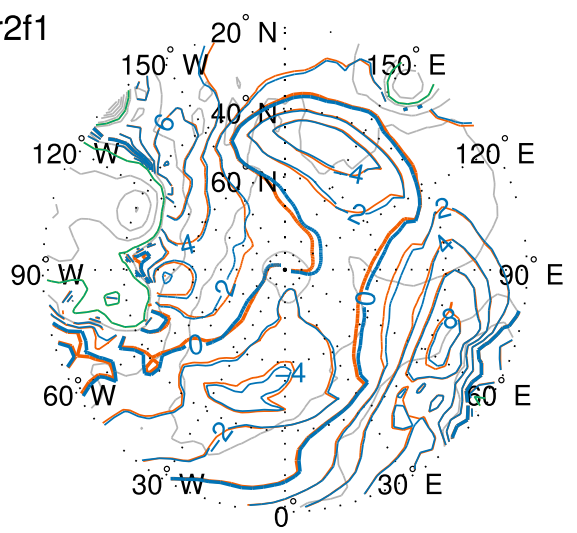

MPREr0.5f1

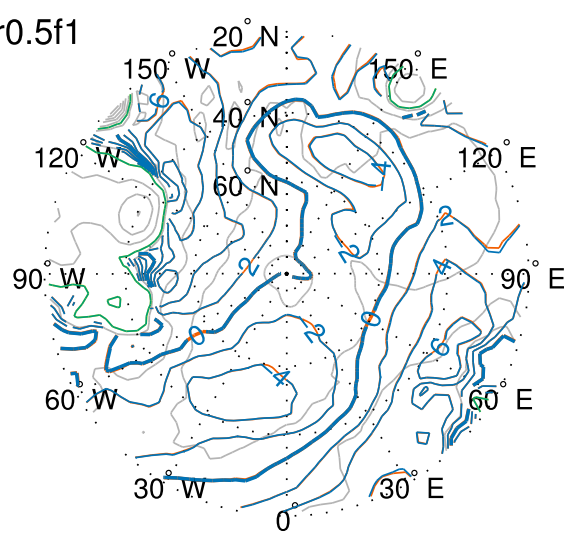

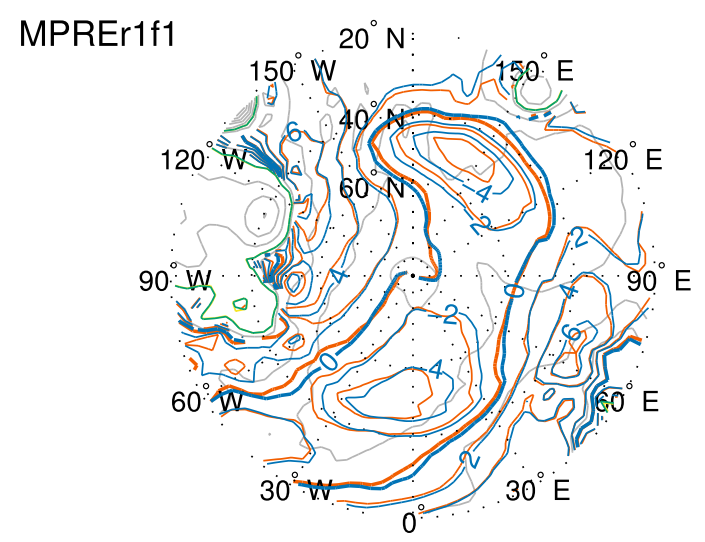
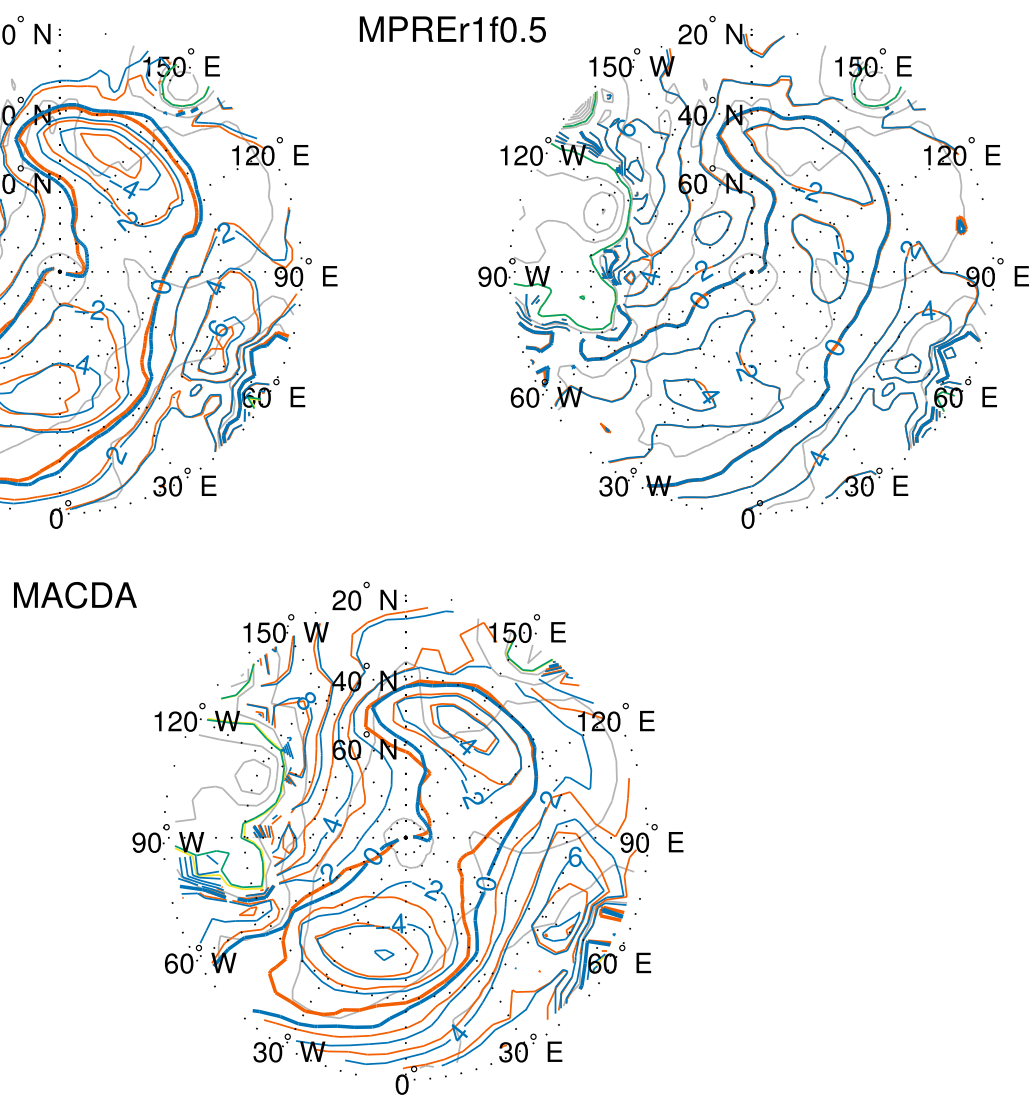

FIG. 3. Temperature stationary waves at $5.25 \mathrm{hPa}$ for (top),(middle) the MPRE idealized GCM experiments and (bottom) MACDA. Contour spacing is $2 \mathrm{~K}$. Plotting conventions largely parallel those of Fig. 2. The surface topography is shown in gray, with a contour spacing of $2 \mathrm{~km}$. The $5.25-\mathrm{hPa}$ surface intersects the topography in several places-these are marked in green and yellow.

relatively strong eddy activity and maintaining our desired focus on eddies in the lower atmosphere. Contour intervals for the horizontal structure figures vary from panel to panel so as to display the spatial patterns more clearly, deemphasizing the amplitudes that the idealized GCM obviously has problems simulating.

The idealized GCM and MACDA $\overline{T^{\prime 2}}$ fields are notably zonally modulated, as seen in Fig. 6. In MACDA eddy activity is weakened over the northern part of the Tharsis bulge $\left(\sim 120^{\circ} \mathrm{W}\right)$ and less prominently over Arabia Terra/ Terra Sabaea (centered at $\sim 30^{\circ} \mathrm{E}$ ). These gross features are found in all four idealized GCM simulations. However, finer details such as numbers and more precise locations of local maxima are not necessarily well reproduced.

EKE fields are displayed in Fig. 7. Both Mars years of MACDA have their eddy activity maxima in the 

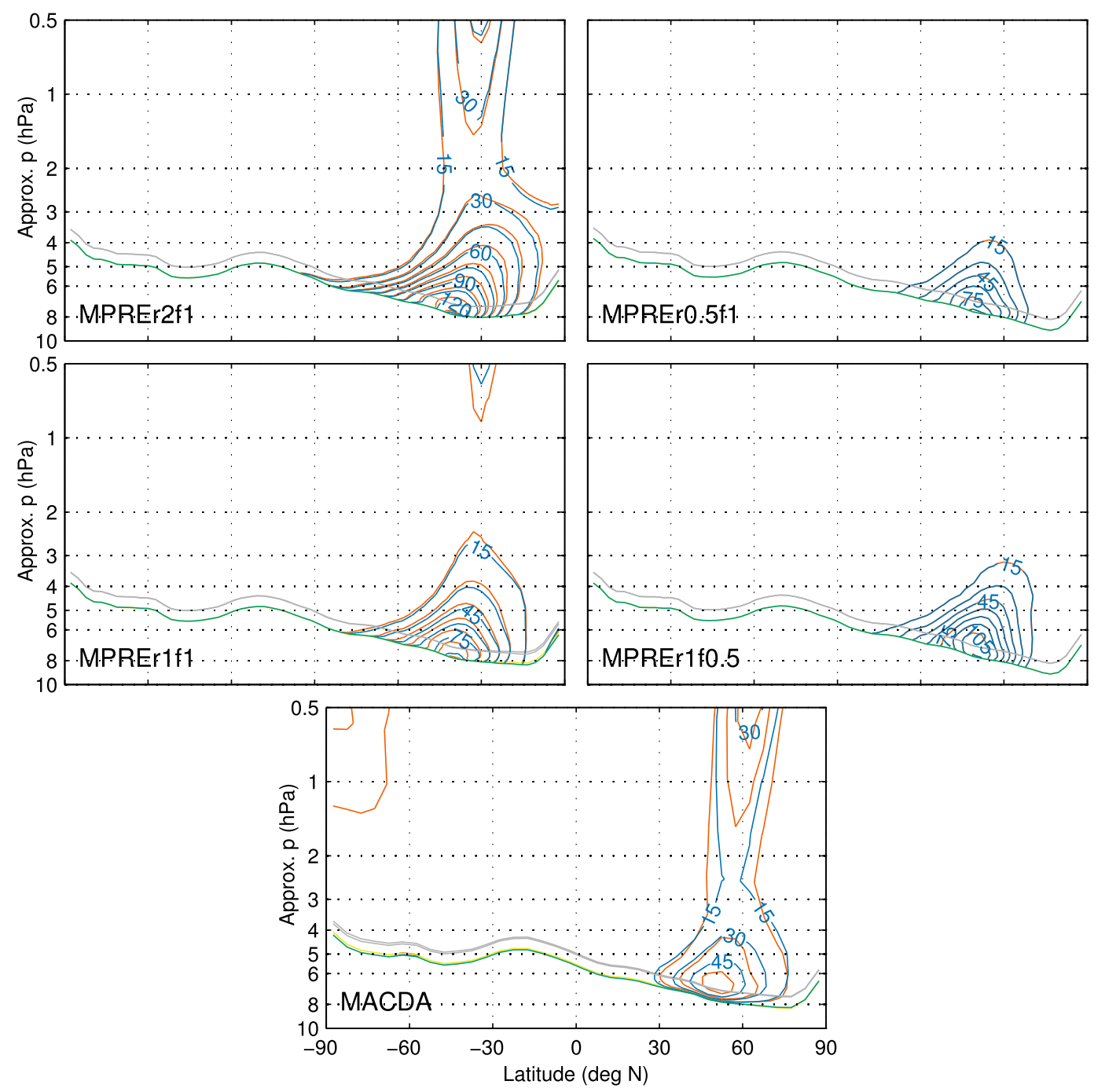

FIG. 4. Zonal-mean $\overline{T^{\prime 2}}$ for the MPRE idealized GCM experiments and MACDA. Contour spacing is $15 \mathrm{~K}^{2}$. The gray lines indicate the level at which the horizontal structure of $\overline{T^{\prime 2}}$ will be evaluated. Other plotting conventions are as in Fig. 2.

hemisphere centered on $180^{\circ}$ longitude, a feature to some degree reproduced by all four idealized GCM simulations. This tendency seems to be captured especially well by MPREr2f1 and MPREr1f1 (at least to the extent that the grossly incorrect maximum at the pole in MPREr2f1 is ignored). In contrast MPREr0.5f1 and MPREr1f0.5 have very weak eddy activity at the highest latitudes, which does not seem to be a feature of MACDA.

\section{4) Overall evaluation of MPRE SIMULATIONS}

To zeroth order, the results presented in this subsection establish that the idealized GCM described in sections $2 b$ and $2 c$ works qualitatively if not quantitatively. The MACDA-based target temperature climatology can be reasonably well reproduced for a range of radiative and frictional damping times. The temperature distribution strongly influences but does not totally determine extratropical wind fields, because thermal wind balance cannot constrain surface winds.

We tested five different combinations of radiative and frictional damping times. One combination $\left(\tau_{R}=\tau_{F}=\right.$ 0.5 sols) virtually eliminated transient eddy activity, but the other four all had at least some success in capturing the primary features of its spatial patterns. However, eddy amplitudes are frequently biased with respect to MACDA. The reasons for this are not clear-perhaps vertical variations in $\tau_{R}$ matter or the Newtonian relaxation-Rayleigh drag physics framework is structurally inadequate. 
TABLE 2. Low-level maximum values of the zonal-mean $\overline{T^{\prime 2}}$ fields for MACDA and the MPRE experiments. For each MPRE experiment, maxima were computed from two separate 270 -sol means. Both such means are listed in the table, separated by commas. For MACDA, the maxima were computed over the appropriate parts of MY24 and 26, respectively. All of the idealized model maxima are located at $\sigma=$ $0.9966,51.6^{\circ} \mathrm{N}$, while the MACDA maxima are at $\sigma=0.8312,52.5^{\circ} \mathrm{N}$.

\begin{tabular}{lc}
\hline \hline & $\overline{T^{\prime 2}}$ maxima $\left(\mathrm{K}^{2}\right)$ \\
\hline MPREr2f1 & $145.2,149.9$ \\
MPREr1f1 & $101.8,109.6$ \\
MPREr0.5f1 & $98.5,98.6$ \\
MPREr1f0.5 & $122.5,122.7$ \\
MACDA & $59.8,51.0$ \\
\hline
\end{tabular}

While no set of damping parameters is superior in all respects, the zonal-mean surface pressures and the meridional extent of the surface westerly jet are fairly clearly more realistic in MPREr2f1 and MPREr1f1 than in MPREr0.5f1 and MPREr1f0.5. In the rest of this paper, we thus primarily use simulations with $\tau_{R}=1$ or 2 sols and $\tau_{F}=1$ sol.

\section{b. Seasonal variations}

Next we evaluate how well the idealized GCM can simulate seasonal changes in transient eddy activity by conducting experiments using the MPAUSE and MPOST target climatologies. We examine the eddies in low-level $\overline{T^{\prime 2}}$ and $\overline{v^{\prime 2}}$ fields-the latter field was not used in the previous subsection, and to ease comparisons between seasons MPRE eddy activity maps are shown here in the same format as their MPAUSE and MPOST counterparts.

Zonal-mean zonal wind fields for the MPAUSE and MPOST idealized GCM simulations are shown in Fig. 8. Agreement between the idealized GCM simulations and MACDA is good in the extratropics, driven by thermal
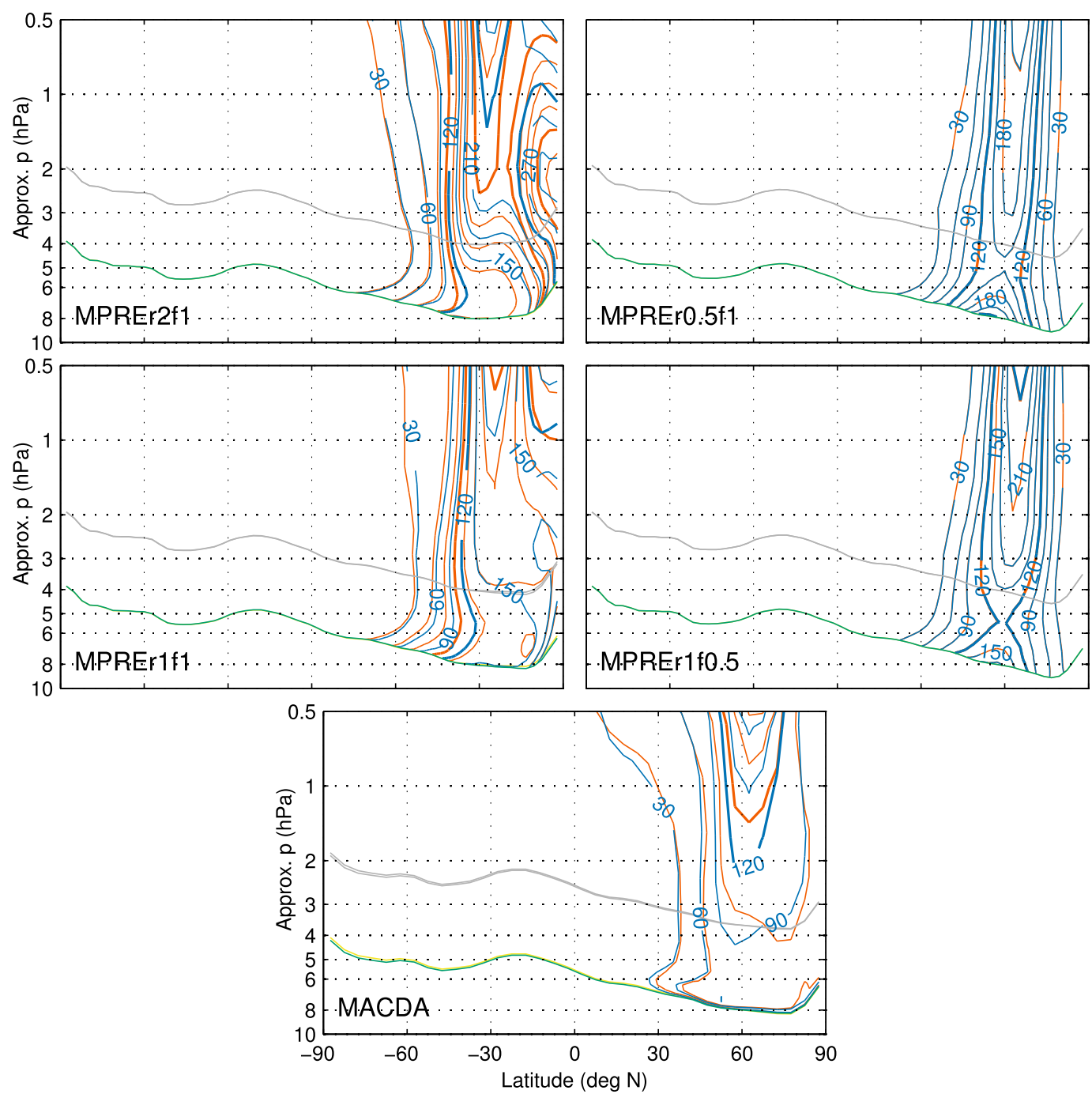

FIG. 5. As in Fig. 4, but for zonal-mean EKE. Contour spacing is $30 \mathrm{~m}^{2} \mathrm{~s}^{-2}$. Contours that are multiples of $120 \mathrm{~m}^{2} \mathrm{~s}^{-2}$ are bolded. The gray lines indicate the level at which the horizontal structure of EKE will be evaluated. 

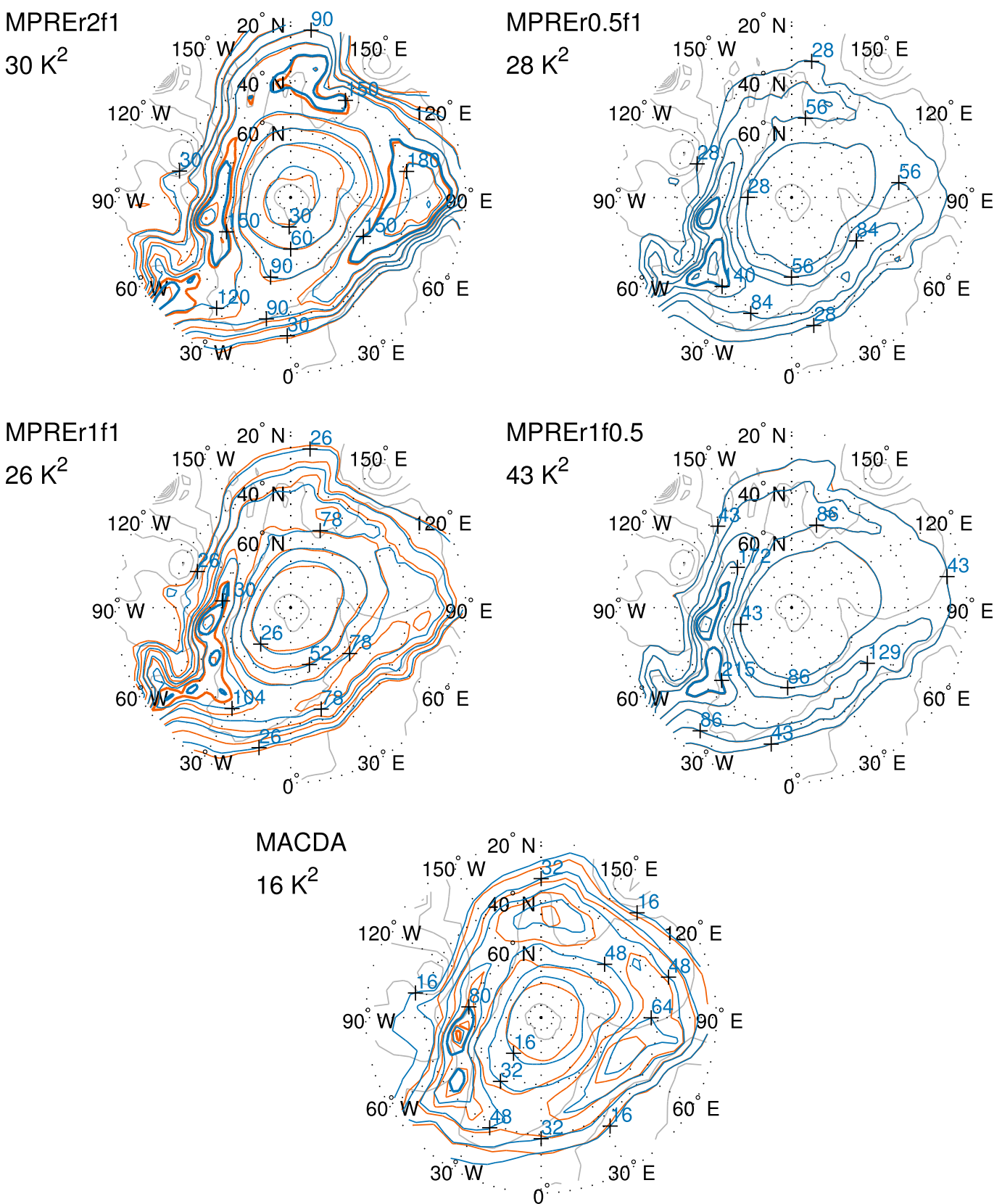

FIG. 6. Plots of $\overline{T^{\prime 2}}$ for the (top),(middle) MPRE idealized GCM experiments and (bottom) MACDA. Data are plotted on $\sigma=0.9046$ for the idealized GCM and $\sigma=0.8996$ for MACDA. Contour spacing for each panel is listed below the associated experiment name. To assist in identifying the locations of maxima, the fifth contour in each panel is bolded. Other plotting conventions are largely as in Fig. 3.

wind balance and the successful reproduction of the MACDA-based target climatologies (Mooring 2016). Although the MPAUSE simulations and MACDA segments share a strong northern westerly jet with their MPRE counterparts, the tropical upper-level jet is much more easterly during MPAUSE. The MPOST Northern Hemisphere jets are notably weaker than those of MPRE and MPAUSE.
The $\overline{T^{\prime 2}}$ and $\overline{v^{\prime 2}}$ fields for experiments with the MPRE, MPAUSE, and MPOST target climatologies are presented as Figs. 9-11. The MPRE $\overline{v^{\prime 2}}$ fields are topographically modulated in a manner similar to their $\overline{T^{\prime 2}}$ counterparts, but wavenumber 3 seems more prominent in the overall pattern. The $\overline{v^{\prime 2}}$ eddies in the idealized GCM simulations are also biased strong relative to MACDA, as measured by area means over $20^{\circ}-90^{\circ}$ and $40^{\circ}-90^{\circ} \mathrm{N}$. 

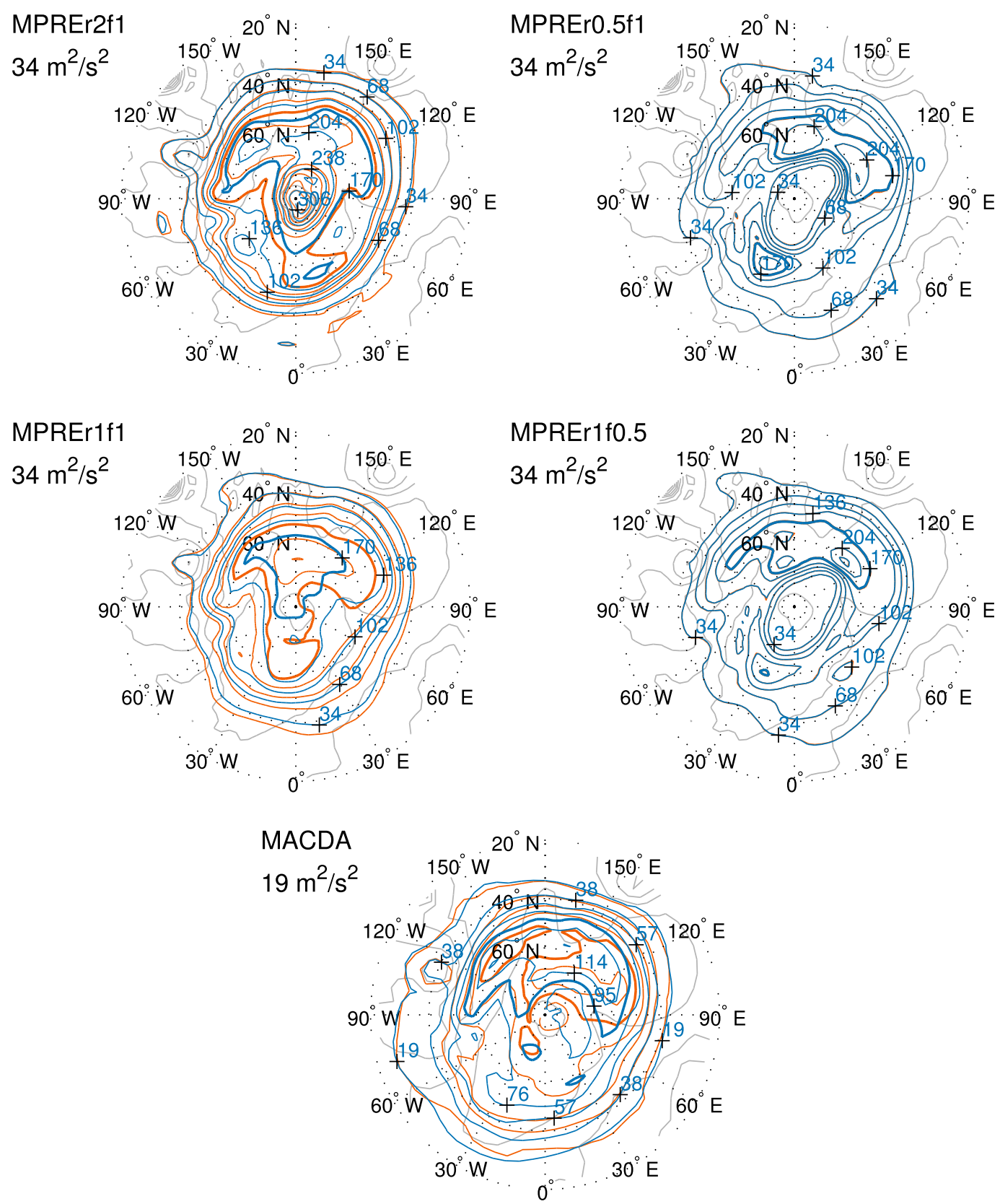

FIG. 7. As in Fig. 6, but for EKE. Data are plotted on $\sigma=0.5025$ for the idealized GCM and $\sigma=0.4553$ for MACDA.

Comparing Figs. 9 and 10, we see that the $\overline{T^{\prime 2}}$ field structures substantially differ-closer to northern winter solstice the region of very weak eddy activity near the pole expands in MACDA, and this phenomenon is successfully reproduced by the idealized GCM simulations. Such a movement of eddy activity away from the pole may also be occurring for $\overline{v^{\prime 2}}$, but its spatial distribution remains dominated by wavenumber 3 . Although the idealized GCM is not very good at reproducing the absolute eddy amplitudes found in MACDA, it remains to be seen whether it can at least qualitatively capture seasonal changes in spatially averaged eddy intensity. Indeed the area averages over $20^{\circ}-90^{\circ}$ and $40^{\circ}-90^{\circ} \mathrm{N}$ of the idealized GCM $\overline{T^{\prime 2}}$ fields shown in Fig. 10 are smaller than their counterparts with the same value of $\tau_{R}$ shown in Fig. 9, although in a fractional sense the weakening is less than found in MACDA. These results are not reproduced for $\overline{v^{\prime 2}}$ - the weakening one would expect from 

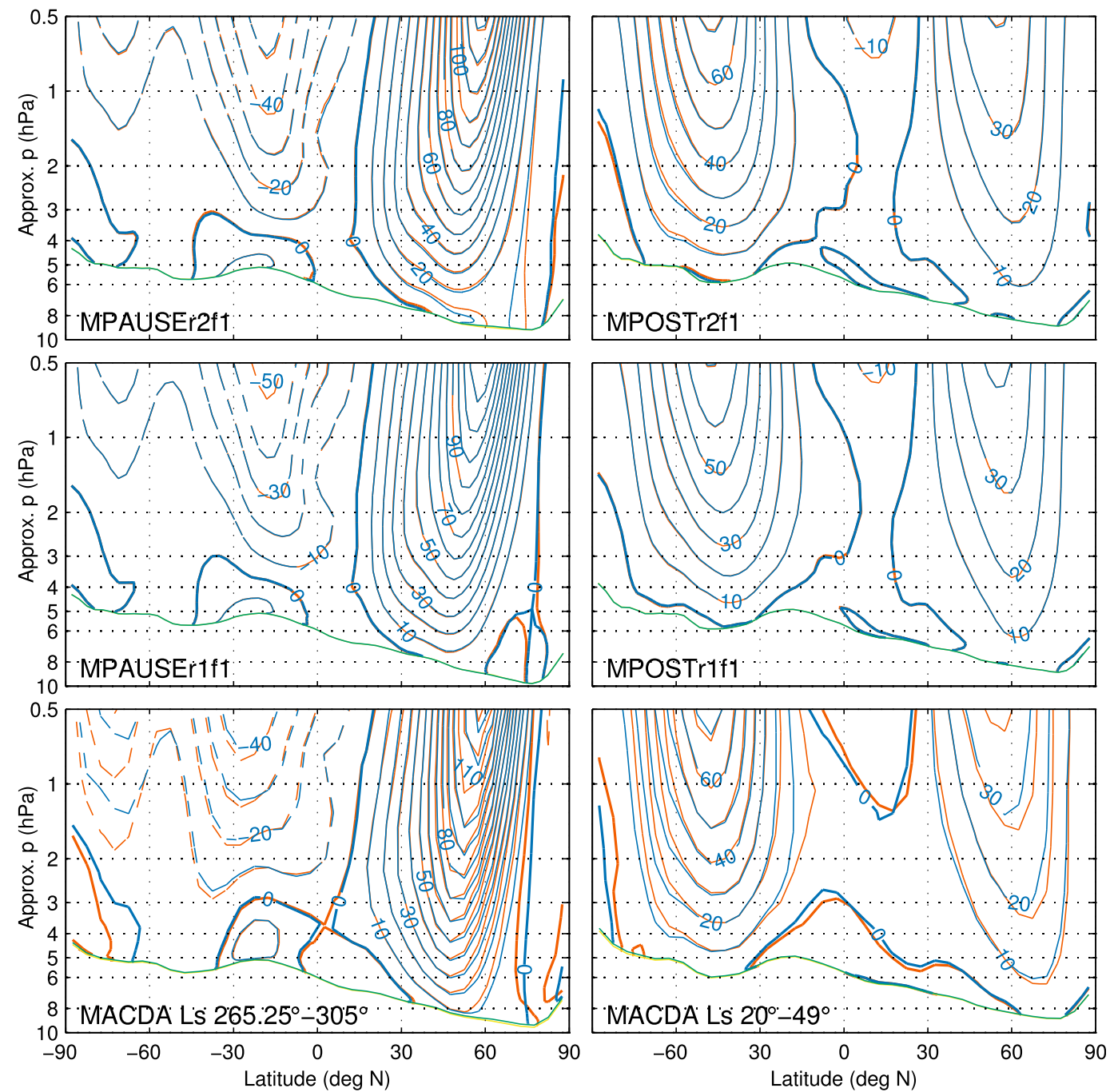

FIG. 8. Time-mean, zonal-mean zonal winds for the (top left),(middle left) MPAUSE and (top right),(middle right) MPOST idealized GCM experiments and (bottom) MACDA. For the idealized GCM simulations, the blue and red lines represent time means over separate 270-sol chunks of data. For MACDA, the blue contours are a time mean over the segment of MY24 or 25 used to define the relevant target climatology and the red contours are for the same $L_{s}$ range in MY26. Other plotting conventions are as in Fig. 2.

MACDA is not robustly detectable in the idealized GCM simulations.

The MPRE and MPAUSE eddy distributions are more similar to each other than they are to those of MPOST (Fig. 11). Relative to the two other seasonal intervals, the MACDA $\overline{T^{\prime 2}}$ eddy activity has largely retreated to north of $\sim 40^{\circ} \mathrm{N}$ and this phenomenon is captured by MPOSTr2f1. It may also be present in MPOSTr1f1, but note the extremely small eddy amplitudes-perhaps the instability of this mean flow is sufficiently weak that it can be stabilized by radiative damping with $\tau_{R}=1 \mathrm{sol}$. The MACDA $\overline{v^{\prime 2}}$ fields for both MY25 and 26 have two prominent local maxima that are present in approximately the correct places in MPOSTr2f1. However, the
MPOSTr1f1 $\overline{v^{\prime 2}}$ field bears little resemblance to that of MACDA. Even for MPOSTr2f1, the eddies are clearly weaker than in MACDA as measured by area means over $20^{\circ}-90^{\circ}$ and $40^{\circ}-90^{\circ} \mathrm{N}$. This is a departure from the strength bias found in the MPRE and MPAUSE simulations, which had eddies that were robustly too strong.

The dynamics of Martian eddy amplitude seasonality have recently been addressed by Mulholland et al. (2016). They argue that the seasonal cycle in eddy strength is driven by changes in the extratropical maximum near-surface Eady growth rate, based on the similarity of seasonal variations in the two quantities in simulations done with the U.K. Mars GCM. There are a number of obstacles to conducting a strong test of this 

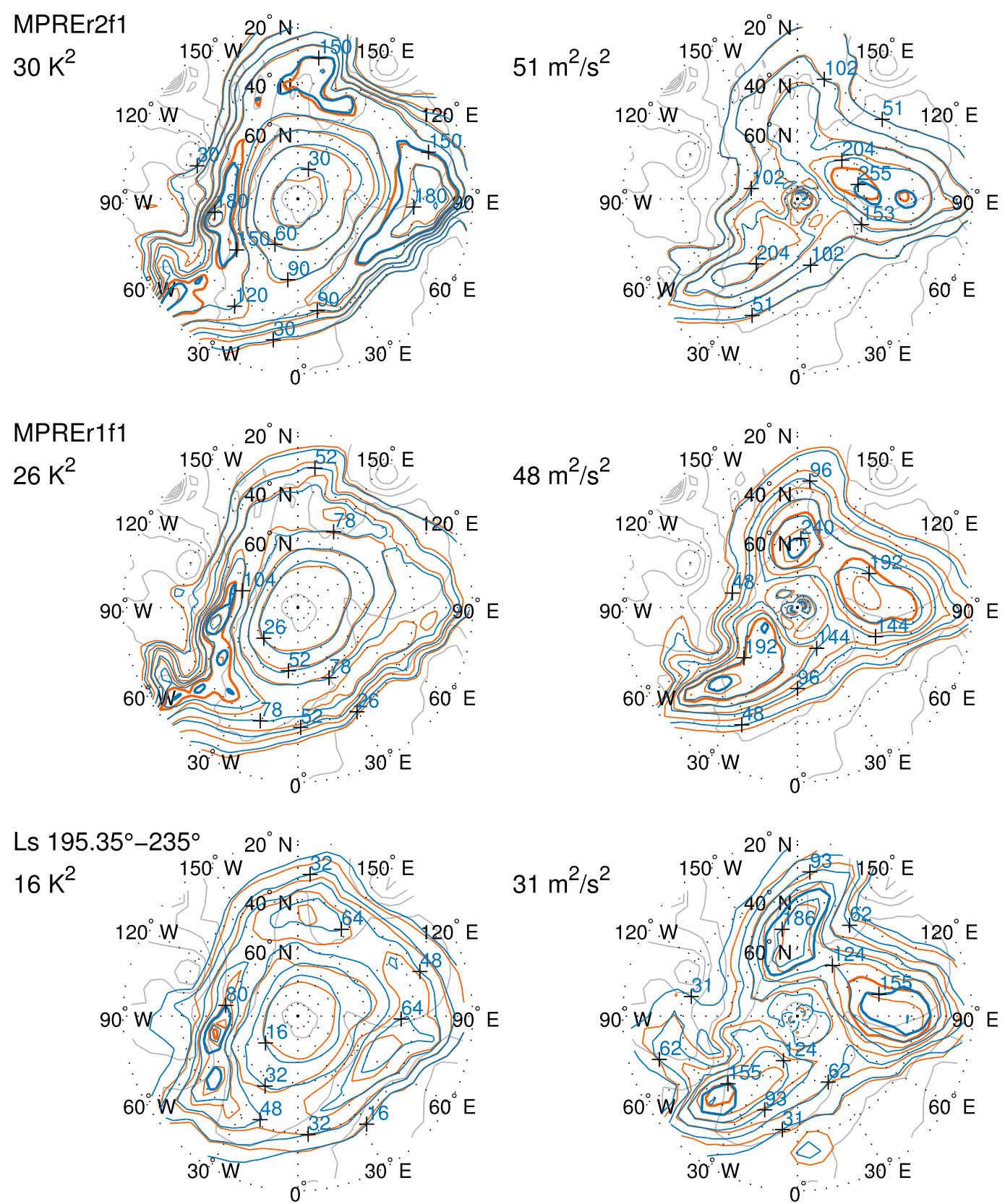

FIG. 9. Plots of (left) $\overline{T^{\prime 2}}$ and (right) $\overline{v^{\prime 2}}$ for (top),(middle) two of the MPRE idealized GCM experiments and (bottom) MACDA. Data are plotted on $\sigma=0.9046$ for the idealized GCM and $\sigma=0.8996$ for MACDA. For each panel, the contour spacing is listed in the upper-left corner. To assist in identifying the locations of maxima, the fifth contour in each panel is bolded. Other plotting conventions are largely as in Fig. 8.

idea using our idealized simulations. First, Mulholland et al.'s (2016) analysis requires data at relatively high seasonal resolution whereas the relevant part of our suite of idealized simulations covers only three fixed seasons. More importantly, the Mulholland et al. (2016) argument is based on output from free-running simulations-the eddy strength-Eady growth rate relationship is less impressive in MACDA itself. This fact does not necessarily invalidate the proposed Eady growth rate mechanism, if one is willing to posit biases in MACDA that render its eddies and mean flow dynamically inconsistent in a way that distorts the relationship between the mean flow and eddy amplitudes. But if this is the case, it is not obvious that idealized simulations with MACDA-based mean flows should be expected to reproduce the MACDA eddy amplitudes. 

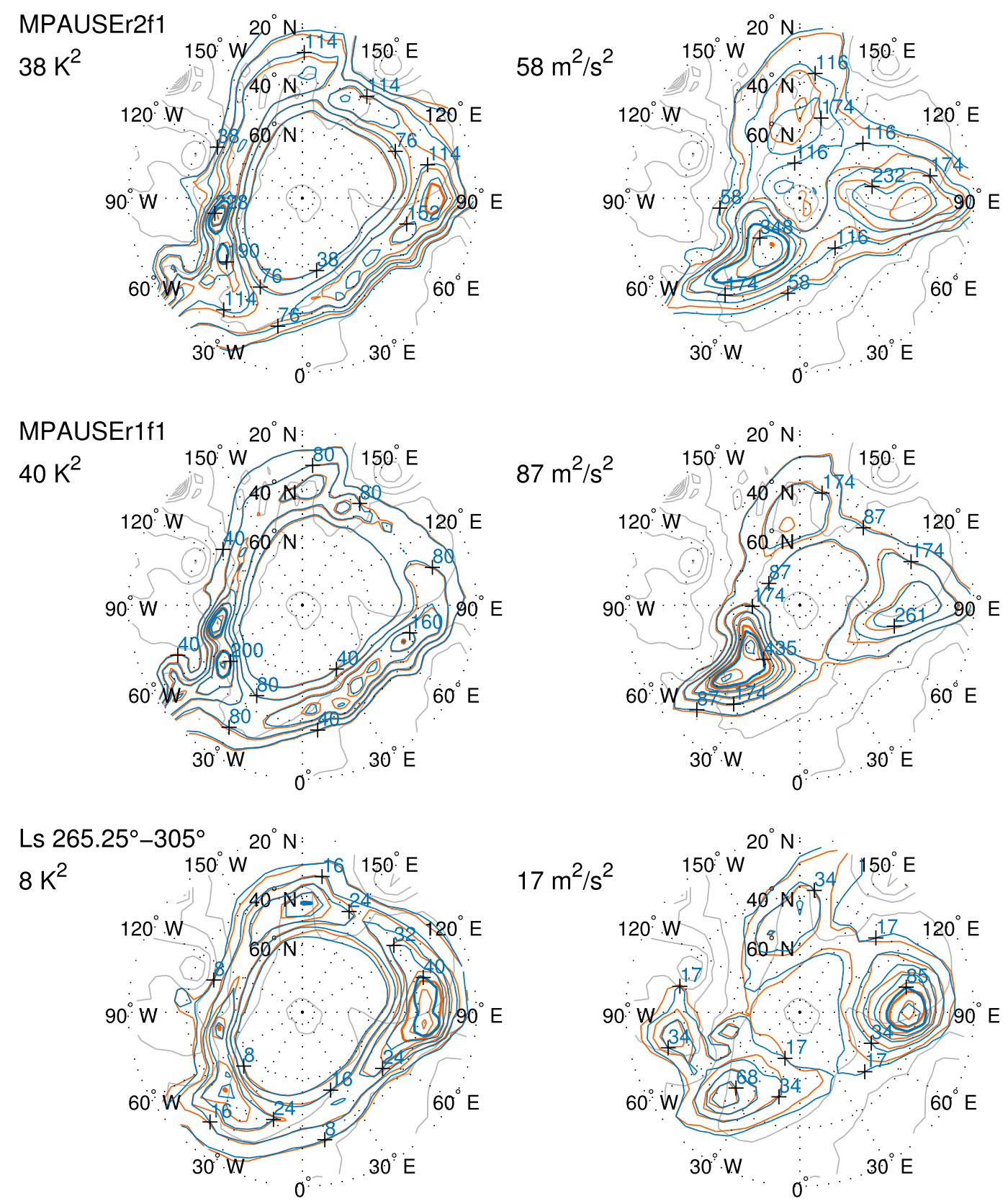

FIG. 10. As in Fig. 9, but for MPAUSE.

Figure 12 shows the relationship between extratropical maximum Eady growth rates and eddy amplitudes in MACDA and the idealized simulations, computed in a manner similar to Mulholland et al. (2016). The pattern noted by Mulholland et al. (2016) in their free-running Mars GCM is not especially evident for MACDA or our idealized simulations, and the idealized GCM simulations poorly reproduce the relevant MACDA maximum Eady growth rates. A more detailed inspection of meridionally resolved Eady growth rates indicates that not merely the amplitudes but also the spatial structures of MACDA zonal-mean Eady growth rates are not well portrayed by the idealized model (not shown). Furthermore, examination of extratropical maximum Eady growth rates and eddy amplitudes for the MPRE simulations makes clear that amplitudes can vary widely in response to changes in the radiative and frictional damping times while growth rates remain nearly fixed (not shown).

Overall, we conclude that the idealized GCM is capable of reproducing some aspects of the seasonal cycle 

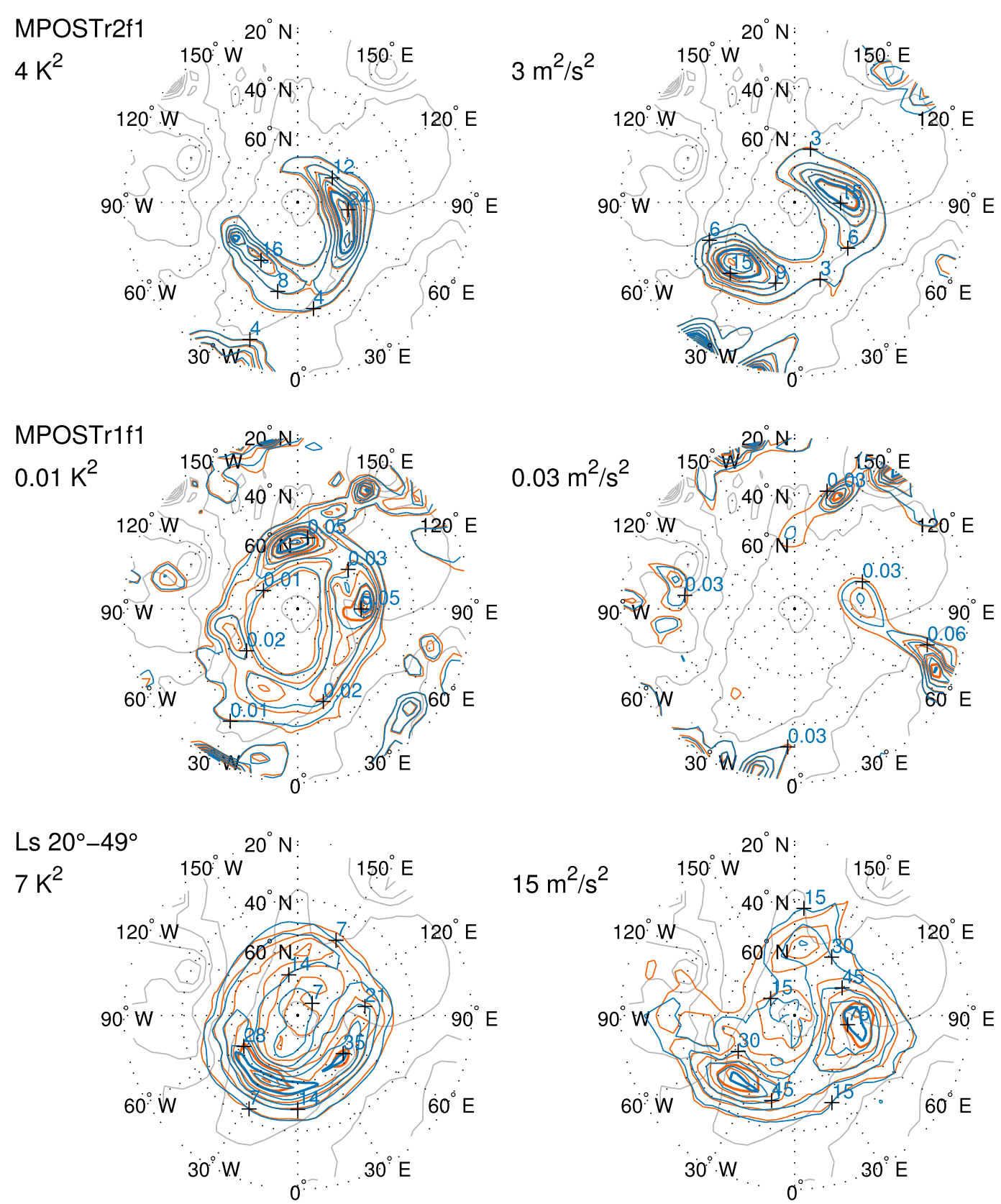

FIG. 11. As in Fig. 9, but for MPOST.

of Northern Hemisphere transient eddy activity found in MACDA. This suggests that the gross features of the spatial distributions of low-level $\overline{T^{\prime 2}}$ and $\overline{v^{\prime 2}}$ in the Martian atmosphere are indeed influenced by the seasonally varying mean flow. Unfortunately eddy amplitudes remain unrealistic - not only are they inaccurate in an absolute sense for individual seasons, the qualitative nature of the seasonal changes seen in MACDA (a prominent drop in area mean eddy intensities from MPRE to MPAUSE, with MPAUSE and MPOST values relatively similar) is not reproduced. Instead the most prominent drop occurs between MPAUSE and MPOST, even with $\tau_{R}=2$ sols. The eddies have become so weak in MPOSTr1f1 (and their spatial distributions so poor) that we ascribe little physical significance to the output of this simulation.

\section{c. Mars GCM eddy wavenumber bias}

Finally, we investigate whether idealized GCM simulations with the MPRE and GPRE target climatologies 


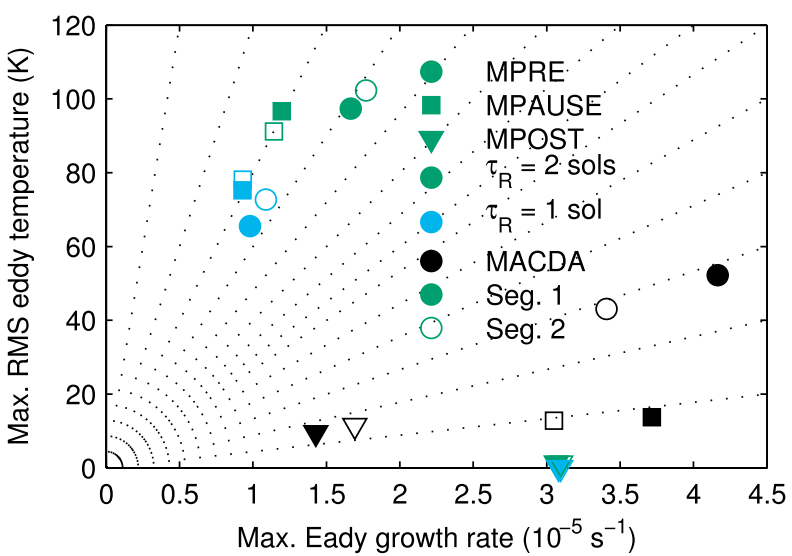

FIG. 12. Relationship between Eady growth rate and $\overline{T^{\prime 2}}$ in MACDA and the idealized GCM simulations, computed similar to Mulholland et al. (2016). The Eady growth rate is defined as $\sigma_{\text {Eady }}=0.31(f / N) \partial U / \partial z$, with $f, N$, and $\partial U / \partial z$ the Coriolis parameter, buoyancy frequency, and vertical shear of zonal-mean zonal wind, respectively. Both $\sigma_{\text {Eady }}$ and $\overline{T^{\prime 2}}$ are characterized by their maxima over $30^{\circ}-90^{\circ} \mathrm{N}$; $\sigma_{\text {Eady }}$ is evaluated over $\sigma=0.97-0.99$, $\sim 200 \mathrm{~m}$ above ground, and $T^{\prime 2}$ is evaluated on $\sigma=0.7310(\sim 3.1 \mathrm{~km})$ for MACDA and $\sigma=0.7483(\sim 2.9 \mathrm{~km})$ for the idealized GCM. The shape of each marker indicates the season, while the color indicates the radiative damping time (if the data are from an idealized GCM simulation) or that the data are from MACDA. To evaluate the sensitivity of the results to internal variability, calculations were done twice for each season-dataset combination. The two separate calculations are denoted using filled and unfilled markers. For the idealized GCM simulations, they are based on two nonoverlapping 270-sol segments. For MACDA, segment 1 is based on the part of MY24 or 25 used to define the target climatology and segment 2 is based on MY26.

capture a bias in the characteristic zonal wavenumber of transient eddies found in the GFDL Mars GCM simulation described in section 2a. Kavulich et al. (2013) showed that for this Mars GCM transient eddy activity in the 5.7-hPa temperature field is clearly concentrated in zonal wavenumber 2 during $L_{s} \approx 195^{\circ}-235^{\circ}$, while eddy activity is more evenly distributed over wavenumbers 1-3 for the same $L_{s}$ range of MACDA MY24.

Figure 13 provides information about the fraction of this time interval for which each zonal wave has the largest amplitude. Wavenumber 2 is largest for a greater fraction of the time in the GFDL Mars GCM than in MACDA, a phenomenon that is particularly visible when eddies are examined in the meridional wind field. The magnitude of the difference is clearly larger than can be explained by internal variability, as it is repeatable across 2 Mars years. Note that while both Mars years of the GFDL Mars GCM simulation are forced by the same (MY24-based) dust scenario, the MACDA MY26 dust is based on actual MY26 observations. Thus a comparison of the MACDA MY24 and 26 wavenumber distributions is more likely to be an overestimate than an underestimate of the amount of variability possible in the actual atmosphere under the constraint of a MY24-like dust field.

Since we hypothesize that the transient eddy activity differences between MACDA and the GFDL Mars GCM are driven by mean flow differences, we examined several time-mean fields from the Mars GCM simulation. The zonal-mean zonal wind and the temperature stationary wave at $56^{\circ} \mathrm{N}$ are shown in Fig. 14 . The zonal jet of the GFDL Mars GCM has a different structure than that of MACDA, appearing broader and weaker near the jet core. The zonal-vertical temperature stationary wave section shows that the GFDL Mars GCM wave is of similar vertical structure to its MACDA counterpart but is substantially weaker. Time-mean meridional winds and temperature stationary waves were also examined at 5.25 and $3 \mathrm{hPa}$, respectively (Mooring 2016). The Mars GCM's 5.25-hPa meridional wind field has a similar spatial structure to that of MACDA and the 3-hPa temperature stationary wave differs conspicuously in amplitude from its MACDA counterpart, as one would expect from the zonal-vertical stationary wave. The temperature stationary wave may also be phase shifted relative to MACDA, with the GFDL Mars GCM wave extrema located farther east.

The same fields as shown in Fig. 14 are presented for the idealized GCM simulations with the GPRE target climatology in Fig. 15. There are three such simulations with different values of $\tau_{R}$ (GPREr2f1, GPREr1f1, and GPREr0.5f1). The GPRE simulations arguably reproduce the greater width and relative weakness of the GFDL Mars GCM zonal jet relative to MACDA, although there is a suggestion of splitting in the idealized GCM jets not found in the GFDL Mars GCM. Agreement of the GPRE idealized GCM temperature stationary waves with the GFDL Mars GCM is not especially impressive, although the local maximum near Tharsis $\left(\sim 260^{\circ} \mathrm{E}\right)$ and the local minimum to the west are in roughly the correct places. The GPRE idealized GCM simulations also capture the fact that the temperature stationary wave is weaker in the GFDL Mars GCM than in MACDA.

A more quantitative demonstration of the idealized GCM's ability to reproduce differences between the MACDA and GFDL Mars GCM mean states is provided by Fig. 16, which shows RMS differences between the time-mean temperature fields of six MPRE and GPRE idealized GCM simulations and the MPRE and GPRE target climatologies. The RMS errors reveal that the temperature fields from each set of simulations (MPRE and GPRE) are closer to the target climatology the simulations are seeking to replicate than they are to the other target climatology. In this sense, the 

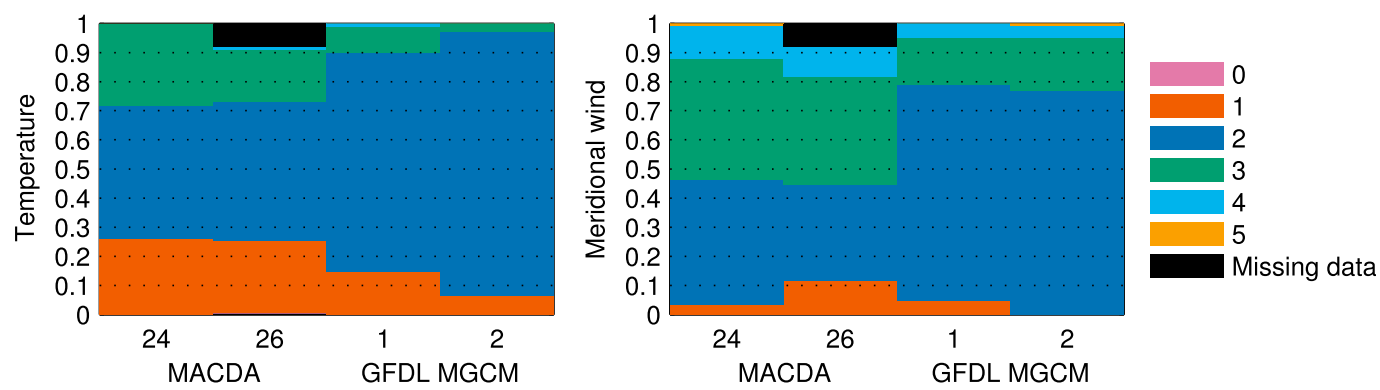

FIG. 13. Fractions of the 62 -sol $L_{s} \approx 195^{\circ}-235^{\circ}$ interval dominated by different zonal wavenumbers in MACDA and the GFDL Mars GCM. To create these graphs, time-dependent transient eddy wavenumber spectra were computed at $\sigma=0.8996,57.5^{\circ} \mathrm{N}$ for MACDA and on a model level with $\sigma \approx 0.90,56^{\circ} \mathrm{N}$ for the GFDL Mars GCM. The largest-amplitude wave for each time step was then identified and used to compute the number of time steps for which each wave had the largest amplitude. Wavenumber 0 is included in the legend because it is dominant for a single time step of the MACDA MY26 eddy temperature spectrum.

idealized GCM simulations successfully capture the distinct MACDA and GFDL Mars GCM time-mean temperature fields. Although Fig. 16 shows RMS errors computed for only a single three-dimensional region, the same general result is obtained for other regions examined (not shown).

We present the wavenumber dominance information for the idealized GCM simulations in Fig. 17. For radiative damping times of 1 and 2 sols, wavenumber 2 is more frequently dominant in simulations with the GPRE target climatology than in simulations with the MPRE target climatology. When the radiative damping time is reduced to 0.5 sols, a single wavenumber overwhelmingly dominates each simulation in an unrealistic manner. Once again the amplitudes of the eddies are not necessarily realistic - in fact, the eddy wavenumber information is presented in this format precisely because the quantification technique used in Figs. 13 and 17 is relatively insensitive to eddy amplitude errors.
We thus conclude that differences between the mean flows of the GFDL Mars GCM and the actual Martian atmosphere as represented in MACDA are at least part of the cause of the excessive dominance of wavenumber 2 in the Mars GCM's eddy spectra for the $L_{s} \approx$ $195^{\circ}-235^{\circ}$ seasonal interval. This finding is robust to the choice of a 1- or 2-sol radiative damping time, although reducing the damping time to 0.5 sols results in nearly constant dominance of a single zonal wavenumber that is not a good match to the reanalysis or Mars GCM simulation. This suggests that the effective radiative damping time of the actual Martian atmosphere is longer than 0.5 sols, consistent with estimates from radiative transfer calculations (Barnes 1984; Nayvelt et al. 1997; Eckermann et al. 2011).

More significantly, our results also suggest that improving the GFDL Mars GCM's mean flow would be a promising path to making its transient eddies more realistic. Because the mean-state errors are at least
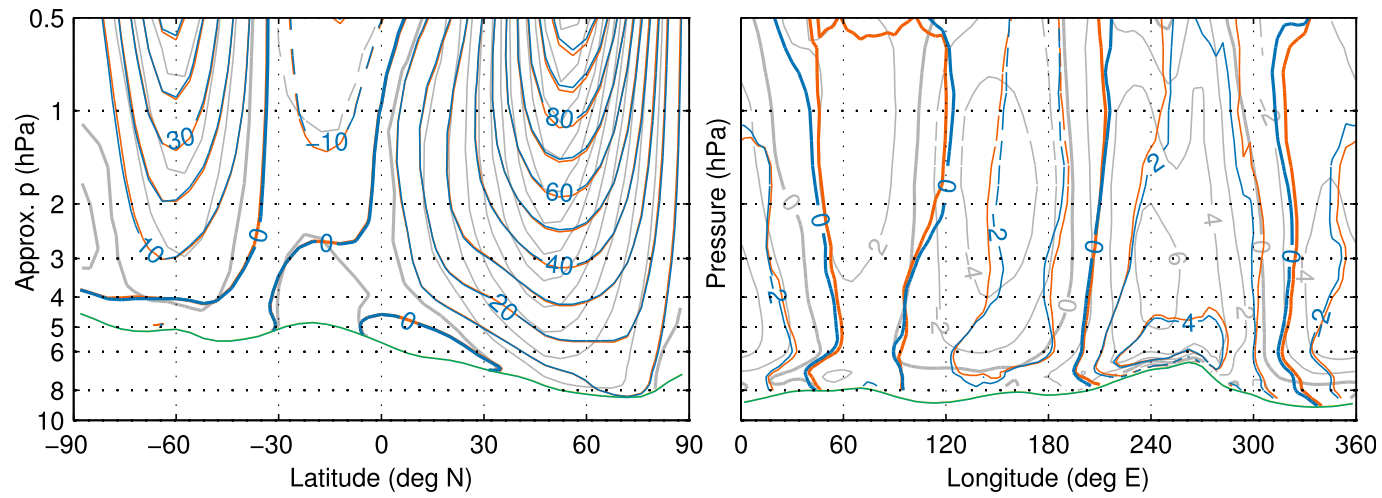

FIG. 14. Time-mean, (left) zonal-mean zonal winds and (right) temperature stationary waves at $56^{\circ} \mathrm{N}$ from the GFDL Mars GCM, with averages over $L_{s}=195.35^{\circ}-234.8^{\circ}$ for MY1 (blue) and MY2 (red). Zonal winds were averaged on model levels but are plotted at approximate pressures, while the temperature stationary waves were computed by first interpolating all of the data to pressure surfaces and then doing suitable averaging. The green and yellow mean surface pressures are for MY1 and 2. Both panels also contain MACDA MY24 data for the same fields (gray). The MACDA temperature stationary wave is from $57.5^{\circ} \mathrm{N}$. 

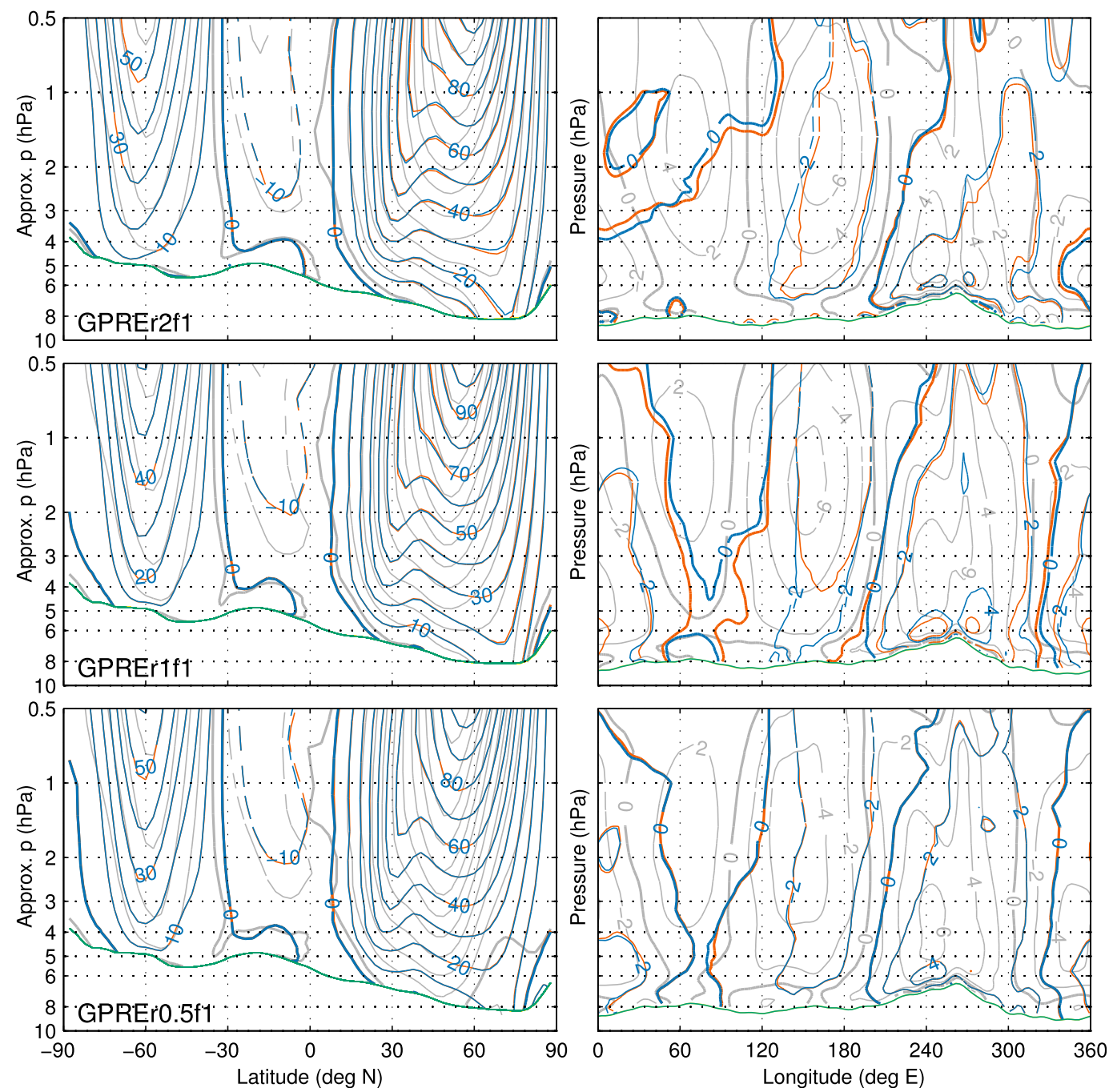

FIG. 15. Time-mean, (left) zonal-mean zonal winds and (right) $57.2^{\circ} \mathrm{N}$ temperature stationary waves for the GPRE idealized GCM simulations. The blue and red contours show separate 270-sol means of output from the GPRE experiments, while the gray contours show 540-sol means from the most comparable MPRE experiments. Other plotting conventions are as in Fig. 14.

partially manifest in the GFDL Mars GCM's temperature field, their most obvious possible source is heating rate errors. Since the relevant clear-sky radiative transfer is relatively well understood, the most likely heating rate error source is weaknesses in the assumed aerosol fields - the dust properties and vertical distribution are difficult to constrain from observations, and the model omits water ice clouds.

\section{Discussion and conclusions}

We have adapted a terrestrial idealized general circulation model with a realistic mean flow to investigate whether the transient eddy statistics of the atmosphere of Mars can be reproduced given an appropriate mean flow and highly simplified assumptions about atmospheric physical processes. Based on extensive tests of the idealized model's ability to simulate one particular seasonal interval $\left(L_{s}=195.35^{\circ}-235^{\circ}\right)$, we find that it is moderately successful in reproducing the threedimensional spatial structures of eddy temperature variance and eddy kinetic energy as portrayed in a reanalysis.

Furthermore, the model has some capacity to simulate the seasonal variation of the horizontal patterns of lowlevel $\overline{T^{\prime 2}}$ and $\overline{v^{\prime 2}}$, including the expansion of the polar region of weak $\overline{T^{\prime 2}}$ from $L_{s}=195.35^{\circ}-235^{\circ}$ to $265.25^{\circ}-$ $305^{\circ}$. It is also able to reproduce the structure of the $L_{s}=20^{\circ}-49^{\circ} \overline{v^{\prime 2}}$ field, albeit only with a radiative damping time of 2 sols. Finally, the idealized model 

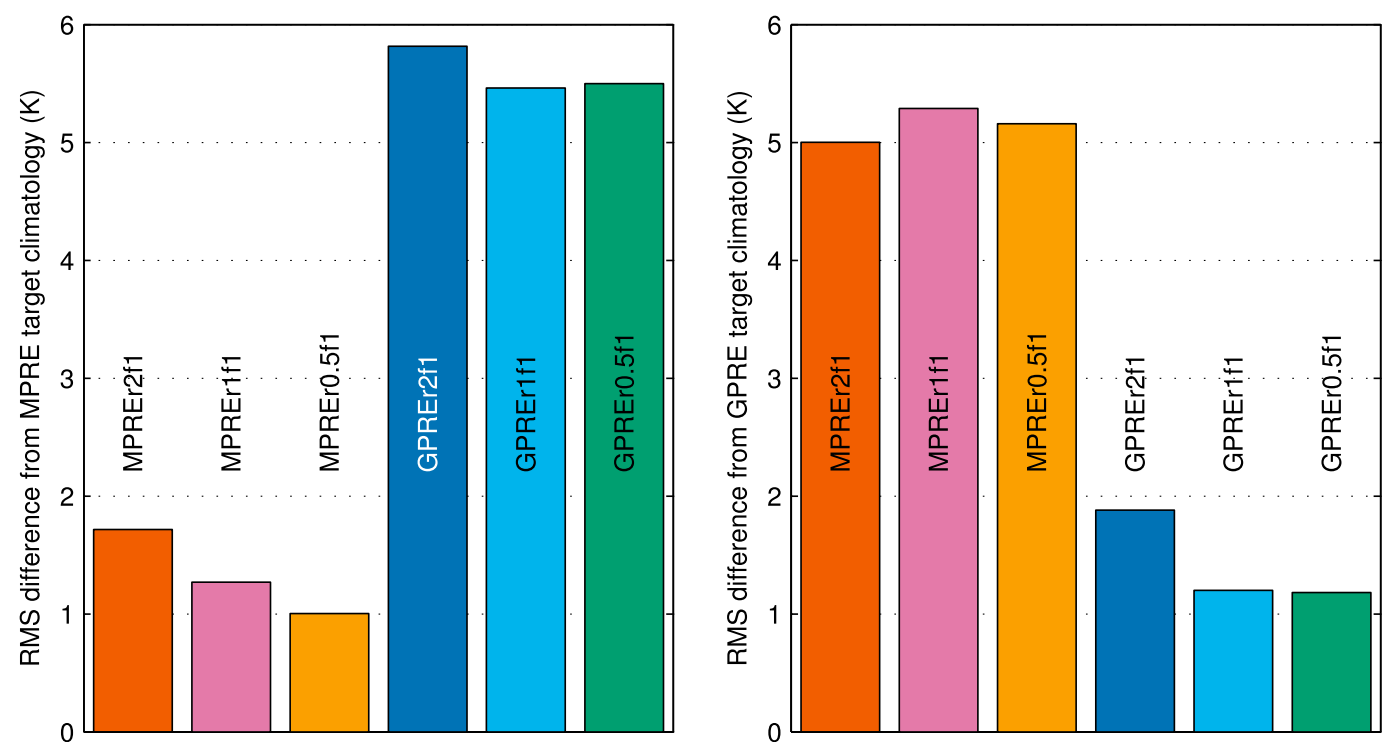

FIG. 16. Mass-weighted RMS differences between the time-mean temperature fields of the MPRE and GPRE idealized GCM simulations and the (left) MPRE and (right) GPRE target climatologies. Each of the six simulations is compared to both target climatologies, yielding the 12 bars shown. The three-dimensional region over which the RMS differences are computed extends over $20^{\circ}-90^{\circ} \mathrm{N}$ and is bounded in the vertical by $0.001 \mathrm{hPa}$ and the spatially varying time-mean surface pressure. Each bar is an average of 10 RMS differences computed from nonoverlapping 60-sol segments of each simulation. The standard deviation of each set of 10 RMS differences is always $<0.15 \mathrm{~K}$-in other words, internal variability is much too small to impact the conclusion that the timemean temperature field for a given simulation is closer to that simulation's target climatology than it is to the other target climatology.

holds promise as a tool for diagnosing errors in Mars GCMs with more realistic parameterizations. When configured to emulate the GFDL Mars GCM, it can in some respects capture this more sophisticated model's bias toward an excessive abundance of wavenumber-2 eddies at one particular season.

By far the most prominent weakness of the idealized model is the unrealistic amplitudes of its transient eddies. Not only do they tend to be biased in an absolute sense (often too strong), their seasonal variation is not well reproduced. Unfortunately, we were not able to clearly link the eddy seasonal cycle errors in our simulations to the related hypotheses of Mulholland et al. (2016).

There are many possibilities for further studies of Martian transient eddies with the idealized GCM. Even using the existing suite of simulations, there is likely scope for additional diagnostic work that explicitly uses more advanced dynamical concepts such as potential vorticity (e.g., Banfield et al. 2004; Seviour et al. 2017). Furthermore, the same basic analyses presented here could be repeated for the Southern Hemisphere.

The ability of the model to reproduce the eddy response to smaller seasonal or subseasonal changes in the mean state could also be explored-for example, we attempted to investigate a transition between different dominant zonal wavenumbers in MACDA during MY24 $L_{s}=222^{\circ}-238^{\circ}$, with ambiguous results. Simulations of more seasonal intervals might also be of use for further investigating Mulholland et al.'s (2016) solstitial pause ideas, particularly if the idealized simulations' mean flows are based on a free-running fullphysics Mars GCM in which an Eady growth rate-eddy amplitude relationship clearly exists.

All of the target climatologies used in this paper have been simple time averages of MACDA or GFDL Mars GCM output, but more complex and arbitrary climatologies might shed light on the underlying dynamics. For example, the target stationary waves could be varied at fixed zonal-mean temperature in a manner similar to the terrestrial study of Chang and Zurita-Gotor (2007). This might clarify the source of differences between MACDA and the GFDL Mars GCM, or enable further testing of Hollingsworth et al.'s (1996) proposed mechanism for creating the horizontal variations of eddy intensity.

Finally, the use of slightly more complex physical parameterizations might enable improvements in the realism of the eddies. This could be particularly helpful for investigations of the seasonal cycle of eddy amplitude, and a first step in this direction may be to objectively estimate a spatially variable radiative damping 

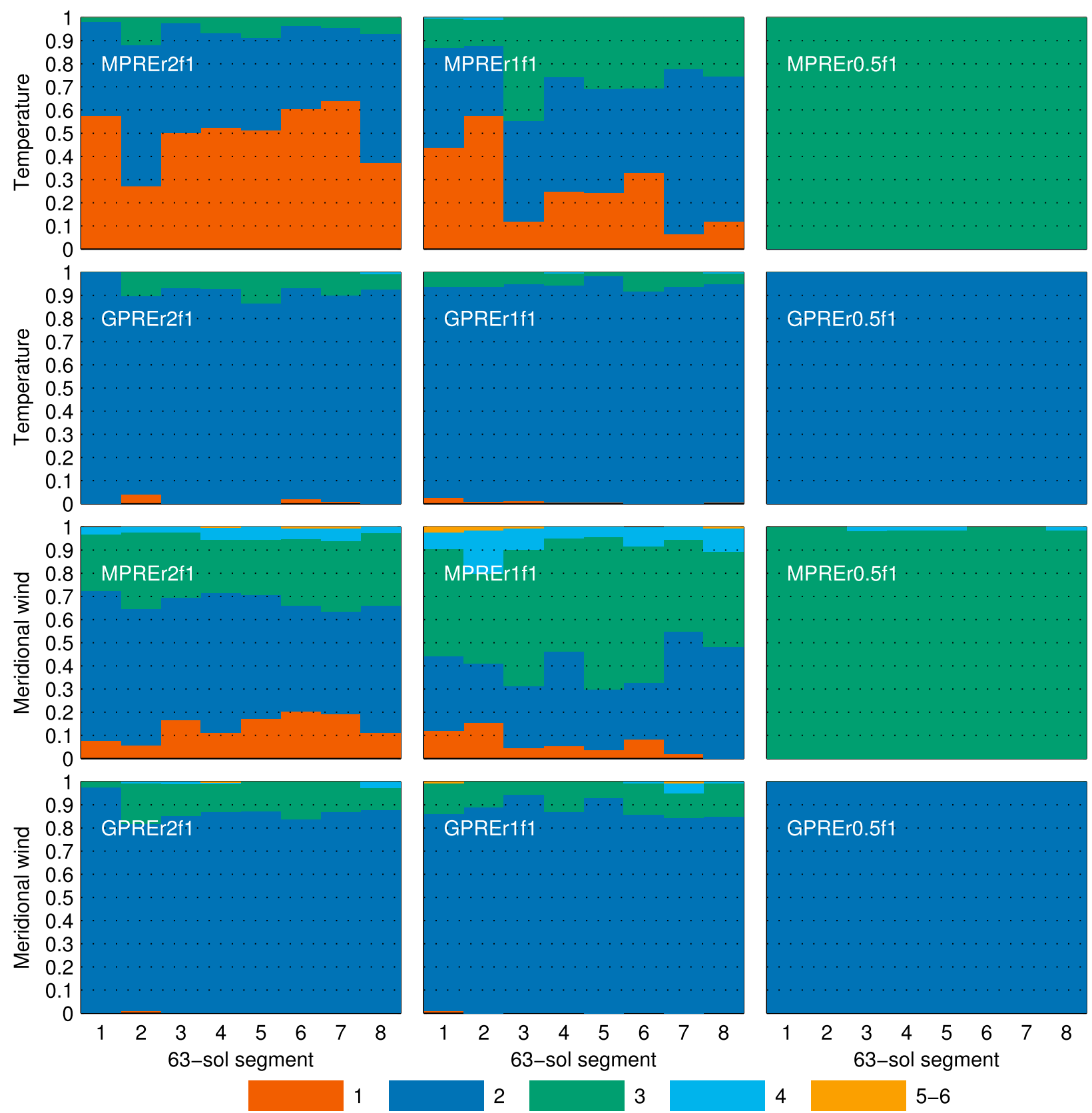

FIG. 17. As in Fig. 13, but for six idealized GCM simulations with (first row),(third row) MPRE and (second row),(fourth row) GPRE target climatologies. The underlying spectra are computed from data at $57.2^{\circ} \mathrm{N}, \sigma=0.9046$. Presenting the results for each simulation in eight 63-sol segments confirms that the key qualitative results are not sensitive to internal variability. Nor does the drop in the frequency of wavenumber-1 dominance across multiple 63-sol segments of MPREr1f1 portend its total disappearance-the apparent downward trend does not continue in an extension of the simulation.

time for use in the model via a technique similar to that of Hitchcock et al. (2010).

Acknowledgments. We thank Luca Montabone for supplying a topographic map on the same horizontal grid as the MACDA dataset. MACDA itself is available from the British Atmospheric Data Centre. Copies of the output of the GFDL Mars GCM simulation and the various idealized model simulations used in this study are available from TAM upon request. TAM was supported by the National Science Foundation Graduate Research Fellowship Program under Grant DGE 1148900, the National Oceanic and Atmospheric Administration, U.S. Department of Commerce under Award NA14OAR4320106, 
and the David and Lucile Packard Foundation. Comments from three anonymous reviewers substantially improved the paper.

\section{REFERENCES}

Allison, M., and M. McEwen, 2000: A post-Pathfinder evaluation of areocentric solar coordinates with improved timing recipes for Mars seasonal/diurnal climate studies. Planet. Space Sci., 48, 215-235, https://doi.org/10.1016/S0032-0633(99)00092-6.

Banfield, D., B. J. Conrath, M. D. Smith, P. R. Christensen, and R. J. Wilson, 2003: Forced waves in the Martian atmosphere from MGS TES nadir data. Icarus, 161, 319-345, https:// doi.org/10.1016/S0019-1035(02)00044-1.

,-- , P. J. Gierasch, R. J. Wilson, and M. D. Smith, 2004: Traveling waves in the Martian atmosphere from MGS TES nadir data. Icarus, 170, 365-403, https://doi.org/10.1016/ j.icarus.2004.03.015.

Barnes, J. R., 1980: Time spectral analysis of midlatitude disturbances in the Martian atmosphere. J. Atmos. Sci., 37, 2002-2015, https:// doi.org/10.1175/1520-0469(1980)037<2002:TSAOMD>2.0.CO;2. , 1984: Linear baroclinic instability in the Martian atmosphere. J. Atmos. Sci., 41, 1536-1550, https://doi.org/10.1175/15200469(1984)041<1536:LBIITM>2.0.CO;2.

— J. B. Pollack, R. M. Haberle, C. B. Leovy, R. W. Zurek, H. Lee, and J. Schaeffer, 1993: Mars atmospheric dynamics as simulated by the NASA Ames general circulation model: 2 . Transient baroclinic eddies. J. Geophys. Res., 98, 3125-3148, https://doi.org/10.1029/92JE02935.

Blackmon, M. L., and N.-C. Lau, 1980: Regional characteristics of the Northern Hemisphere wintertime circulation: A comparison of the simulation of a GFDL general circulation model with observations. J. Atmos. Sci., 37, 497-514, https://doi.org/ 10.1175/1520-0469(1980)037<0497:RCOTNH > 2.0.CO;2.

Blumsack, S. L., and P. J. Gierasch, 1972: Mars: The effects of topography on baroclinic instability. J. Atmos. Sci., 29, 1081-1089, https:// doi.org/10.1175/1520-0469(1972)029<1081:MTEOTO>2.0.CO;2.

Chang, E. K. M., 2006: An idealized nonlinear model of the Northern Hemisphere winter storm tracks. J. Atmos. Sci., 63, 1818-1839, https://doi.org/10.1175/JAS3726.1.

— 2009: Diabatic and orographic forcing of northern winter stationary waves and storm tracks. J. Climate, 22, 670-688, https://doi.org/10.1175/2008JCLI2403.1.

— the Northern Hemisphere storm tracks using idealized nonlinear storm-track models. J. Atmos. Sci., 64, 2309-2331, https://doi.org/10.1175/JAS3957.1.

—- S. Lee, and K. L. Swanson, 2002: Storm track dynamics. J. Climate, 15, 2163-2183, https://doi.org/10.1175/15200442(2002)015<02163:STD>2.0.CO;2.

- Y. Y. Guo, and X. Xia, 2012: CMIP5 multimodel ensemble projection of storm track change under global warming. J. Geophys. Res., 117, D23118, https://doi.org/10.1029/2012JD018578.

Clancy, R. T., B. J. Sandor, M. J. Wolff, P. R. Christensen, M. D. Smith, J. C. Pearl, B. J. Conrath, and R. J. Wilson, 2000: An intercomparison of ground-based millimeter, MGS TES, and Viking atmospheric temperature measurements: Seasonal and interannual variability of temperatures and dust loading in the global Mars atmosphere. J. Geophys. Res., 105, 9553-9571, https://doi.org/10.1029/1999JE001089.

Collins, M., and I. N. James, 1995: Regular baroclinic transient waves in a simplified global circulation model of the Martian atmosphere. J. Geophys. Res., 100, 14421-14432, https:// doi.org/10.1029/95JE01408.

— S. R. Lewis, P. L. Read, and F. Hourdin, 1996: Baroclinic wave transitions in the Martian atmosphere. Icarus, 120, 344 357, https://doi.org/10.1006/icar.1996.0055.

Eckermann, S. D., J. Ma, and X. Zhu, 2011: Scale-dependent infrared radiative damping rates on Mars and their role in the deposition of gravity-wave momentum flux. Icarus, 211, 429442, https://doi.org/10.1016/j.icarus.2010.10.029.

Forget, F., and Coauthors, 1999: Improved general circulation models of the Martian atmosphere from the surface to above 80 km. J. Geophys. Res., 104, 24 155-24175, https://doi.org/ 10.1029/1999JE001025.

Frederiksen, J. S., 1983: Disturbances and eddy fluxes in Northern Hemisphere flows: Instability of three-dimensional January and July flows. J. Atmos. Sci., 40, 836-855, https://doi.org/ 10.1175/1520-0469(1983)040<0836:DAEFIN > 2.0.CO;2.

Gadian, A. M., 1978: The dynamics of and the heat transfer by baroclinic eddies and large-scale stationary topographically forced long waves in the Martian atmosphere. Icarus, 33, 454465, https://doi.org/10.1016/0019-1035(78)90184-7.

Haberle, R. M., H. Houben, J. R. Barnes, and R. E. Young, 1997: A simplified three-dimensional model for Martian climate studies. J. Geophys. Res., 102, 9051-9067, https://doi.org/ 10.1029/97JE00383.

Hall, N. M. J., 2000: A simple GCM based on dry dynamics and constant forcing. J. Atmos. Sci., 57, 1557-1572, https://doi.org/ 10.1175/1520-0469(2000)057<1557:ASGBOD>2.0.CO;2.

Harvey, B. J., L. C. Shaffrey, T. J. Woollings, G. Zappa, and K. I. Hodges, 2012: How large are projected 21st century storm track changes? Geophys. Res. Lett., 39, L18707, https://doi.org/ 10.1029/2012GL052873.

Held, I. M., and M. J. Suarez, 1994: A proposal for the intercomparison of the dynamical cores of atmospheric general circulation models. Bull. Amer. Meteor. Soc., 75, 1825-1830, https://doi.org/10.1175/ 1520-0477(1994)075<1825:APFTIO>2.0.CO;2.

Hinson, D. P., R. J. Wilson, M. D. Smith, and B. J. Conrath, 2003 : Stationary planetary waves in the atmosphere of Mars during southern winter. J. Geophys. Res., 108, 5004, https://doi.org/ 10.1029/2002JE001949.

— , H. Wang, and M. D. Smith, 2012: A multi-year survey of dynamics near the surface in the Northern Hemisphere of Mars: Short-period baroclinic waves and dust storms. Icarus, 219, 307-320, https://doi.org/10.1016/j.icarus.2012.03.001.

Hitchcock, P., T. G. Shepherd, and S. Yoden, 2010: On the approximation of local and linear radiative damping in the middle atmosphere. J. Atmos. Sci., 67, 2070-2085, https:// doi.org/10.1175/2009JAS3286.1.

Hollingsworth, J. L., R. M. Haberle, J. R. Barnes, A. F. C. Bridger, J. B. Pollack, H. Lee, and J. Schaeffer, 1996: Orographic control of storm zones on Mars. Nature, 380, 413-416, https:// doi.org/10.1038/380413a0.

IAU Working Group for Planetary System Nomenclature, 2001: Gazetteer of planetary nomenclature. USGS, accessed 4 September 2016, http://planetarynames.wr.usgs.gov.

James, I. N., and L. J. Gray, 1986: Concerning the effect of surface drag on the circulation of a baroclinic planetary atmosphere. Quart. J. Roy. Meteor. Soc., 112, 1231-1250, https://doi.org/ 10.1002/qj.49711247417.

Kavulich, M. J., Jr., I. Szunyogh, G. Gyarmati, and R. J. Wilson, 2013: Local dynamics of baroclinic waves in the Martian atmosphere. J. Atmos. Sci., 70, 3415-3447, https://doi.org/ 10.1175/JAS-D-12-0262.1. 
Keppenne, C. L., and A. P. Ingersoll, 1995: High-frequency orographically forced variability in a single-layer model of the Martian atmosphere. J. Atmos. Sci., 52, 1949-1958, https:// doi.org/10.1175/1520-0469(1995)052<1949:HFOFVI>2.0.CO;2.

Leovy, C. B., 1969: Mars: Theoretical aspects of meteorology. Appl. Opt., 8, 1279-1286, https://doi.org/10.1364/AO.8.001279.

_ 2001: Weather and climate on Mars. Nature, 412, 245-249, https://doi.org/10.1038/35084192.

_ ment for the atmosphere of Mars. RAND Corporation Research Memo., 52 pp.

_ and _ 1969: Numerical simulation of the atmospheric circulation and climate of Mars. J. Atmos. Sci., 26, 1167-1190, https:// doi.org/10.1175/1520-0469(1969)026<1167:NSOTAC $>2.0 . C O ; 2$.

—, G. A. Briggs, A. T. Young, B. A. Smith, J. B. Pollack, E. N. Shipley, and R. L. Wildey, 1972: The Martian atmosphere: Mariner 9 television experiment progress report. Icarus, 17, 373-393, https://doi.org/10.1016/0019-1035(72)90006-1.

Lewis, S. R., P. L. Read, and M. Collins, 1996: Martian atmospheric data assimilation with a simplified general circulation model: Orbiter and lander networks. Planet. Space Sci., 44, 13951409, https://doi.org/10.1016/S0032-0633(96)00058-X.

,-- , B. J. Conrath, J. C. Pearl, and M. D. Smith, 2007: Assimilation of Thermal Emission Spectrometer atmospheric data during the Mars Global Surveyor aerobraking period. Icarus, 192, 327-347, https://doi.org/10.1016/j.icarus.2007.08.009.

_ - D. P. Mulholland, P. L. Read, L. Montabone, R. J. Wilson, and M. D. Smith, 2016: The solsticial pause on Mars: 1. A planetary wave reanalysis. Icarus, 264, 456-464, https:// doi.org/10.1016/j.icarus.2015.08.039.

Lin, S.-J., 2004: A "vertically Lagrangian" finite-volume dynamical core for global models. Mon. Wea. Rev., 132, 2293-2307, https:// doi.org/10.1175/1520-0493(2004)132<2293:AVLFDC>2.0.CO;2.

LMD Mars Climate Database Team, 2010: Mars date (year and solar longitude) to Earth date conversion. LMD, accessed 4 September 2016, http://www-mars.lmd.jussieu.fr/mars/time/ mars_date_to_earth_date.html.

Lunkeit, F., K. Fraedrich, and S. Bauer, 1998: Storm tracks in a warmer climate: Sensitivity studies with a simplified global circulation model. Climate Dyn., 14, 813-826, https://doi.org/ $10.1007 / \mathrm{s} 003820050257$

Matheson, M., 2000: Baroclinic eddies in the Martian atmosphere: A general circulation model study. M.S. thesis, Dept. of Atmospheric Sciences, Oregon State University, 158 pp., http:// hdl.handle.net/1957/28737.

Mintz, Y., 1961: The general circulation of planetary atmospheres. The Atmospheres of Mars and Venus, W. W. Kellogg and C. Sagan, Eds., National Academy of Sciences, 107-146, https://doi.org/10.17226/12424.

Montabone, L., M. T. Lemmon, M. D. Smith, M. J. Wolff, F. Forget, and E. Millour, 2011: Reconciling dust opacity datasets and building multiannual dust scenarios for Mars atmospheric models. Fourth Int. Workshop on the Mars Atmosphere: Modelling and Observations, Paris, France, CNES and ESA, http://www-mars.lmd.jussieu.fr/paris2011/ abstracts/montabone2_paris2011.pdf.

_ , and Coauthors, 2014: The Mars Analysis Correction Data Assimilation (MACDA) dataset v1.0. Geosci. Data J., 1, 129_ 139, https://doi.org/10.1002/gdj3.13.

Mooring, T. A., 2016: Transient eddies in the Martian atmosphere: Representation in reanalysis and idealized modeling. Ph.D. thesis, Princeton University, 188 pp., http://arks.princeton.edu/ ark:/88435/dsp01z029p721k.
, and R. J. Wilson, 2015: Transient eddies in the MACDA Mars reanalysis. J. Geophys. Res. Planets, 120, 1671-1696, https://doi.org/10.1002/2015JE004824.

Mulholland, D. P., S. R. Lewis, P. L. Read, J.-B. Madeleine, and F. Forget, 2016: The solsticial pause on Mars: 2. Modelling and investigation of causes. Icarus, 264, 465-477, https://doi.org/ 10.1016/j.icarus.2015.08.038.

Nayvelt, L., P. J. Gierasch, and K. H. Cook, 1997: Modeling and observations of Martian stationary waves. J. Atmos. Sci., 54, 986-1013, https://doi.org/10.1175/1520-0469(1997)054<0986: MAOOMS $>2.0$.CO;2.

Petrosyan, A., and Coauthors, 2011: The Martian atmospheric boundary layer. Rev. Geophys., 49, RG3005, https://doi.org/ 10.1029/2010RG000351.

Putman, W. M., and S.-J. Lin, 2007: Finite-volume transport on various cubed-sphere grids. J. Comput. Phys., 227, 55-78, https://doi.org/10.1016/j.jcp.2007.07.022.

Read, P. L., S. R. Lewis, and D. P. Mulholland, 2015: The physics of Martian weather and climate: A review. Rep. Prog. Phys., 78, 125901, https://doi.org/10.1088/0034-4885/78/12/125901.

Ryan, J. A., R. M. Henry, S. L. Hess, C. B. Leovy, J. E. Tillman, and C. Walcek, 1978: Mars meteorology: Three seasons at the surface. Geophys. Res. Lett., 5, 715-718, https:// doi.org/10.1029/GL005i008p00715.

Seviour, W. J. M., D. W. Waugh, and R. K. Scott, 2017: The stability of Mars's annular polar vortex. J. Atmos. Sci., 74, 1533-1547, https://doi.org/10.1175/JAS-D-16-0293.1.

Smith, D., G. Neumann, R. E. Arvidson, E. A. Guinness, and S. Slavney, 2003: Mars Global Surveyor laser altimeter mission experiment gridded data record. NASA Planetary Data System, accessed 6 July 2019, https://pds.nasa.gov/ds-view/pds/ viewDataset.jsp?dsid=MGS-M-MOLA-5-MEGDR-L3-V1.0.

Tanaka, H. L., and M. Arai, 1999: Linear baroclinic instability in the Martian atmosphere: Primitive equation calculations. Earth Planets Space, 51, 225-232, https://doi.org/10.1186/ BF03352226.

USGS Astrogeology Science Center, 2015: Approximate boundaries for Mars regional feature names. USGS Rep., 1 pp., http://planetarynames.wr.usgs.gov/images/mola_ regional_boundaries.pdf.

Valdes, P. J., and B. J. Hoskins, 1988: Baroclinic instability of the zonally averaged flow with boundary layer damping. J. Atmos. Sci., 45, 1584-1593, https://doi.org/10.1175/1520-0469(1988) 045<1584:BIOTZA > 2.0.CO;2.

Wilson, R. J., 2011: Dust cycle modeling with the GFDL Mars general circulation model. Fourth Int. Workshop on the Mars Atmosphere: Modelling and Observations, Paris, France, CNES and ESA, http://www-mars.lmd.jussieu.fr/paris2011/ abstracts/wilson_rj3_paris2011.pdf.

Wu, Z., and T. Reichler, 2018: Towards a more Earth-like circulation in idealized models. J. Adv. Model. Earth Syst., 10, 1458-1469, https://doi.org/10.1029/2018MS001356.

Zalucha, A. M., and A. A. S. Gulbis, 2012: Comparison of a simple 2-D Pluto general circulation model with stellar occultation light curves and implications for atmospheric circulation. J. Geophys. Res., 117, E05002, https://doi.org/10.1029/ 2011JE003957.

Zhao, M., I. M. Held, S.-J. Lin, and G. A. Vecchi, 2009: Simulations of global hurricane climatology, interannual variability, and response to global warming using a $50-\mathrm{km}$ resolution GCM. J. Climate, 22, 6653-6678, https://doi.org/10.1175/ 2009JCLI3049.1. 